\title{
Stable model order reduction for time-domain exterior vibro-acoustic finite element simulations
}

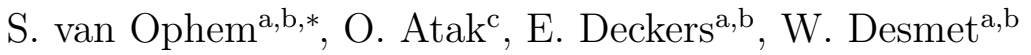 \\ ${ }^{a}$ KU Leuven, Department of Mechanical Engineering, Celestijnenlaan 300 B, B-3001, \\ Heverlee, Belgium \\ ${ }^{b}$ Member of Flanders Make \\ ${ }^{c}$ Siemens Industry Software NV, Interleuvenlaan 68, B-3001, Leuven, Belgium
}

\begin{abstract}
This paper presents a novel method that enables model order reduction of a fully-coupled, exterior vibro-acoustic finite element model for time domain simulations. The method preserves the stability of the full model and reduces the amount of degrees of freedom significantly, with only a moderate amount of calculation complexity. Infinite elements are used on the finite element boundary to satisfy the Sommerfeld radiation condition. Two different strategies to calculate the reduced order model are compared. The first strategy works with a split reduced basis and can be applied on any fully stable model. The second strategy starts from a modified Everstine formulation and directly builds a reduced basis from the full model, leading to more compact reduced order models. Furthermore, a method is derived to perform explicit time integration on the reduced system, while avoiding the inversion of the mass matrix, which might not be possible due to the presence of the infinite elements. Also this method is shown to preserve the stability of the model and a computationally efficient way for implementation of the method is discussed. The effectiveness of the novel methodology is demonstrated with two numerical models.
\end{abstract}

Keywords: Model order reduction, Exterior vibro-acoustics, Time domain, Finite element method, Infinite elements

\section{Introduction}

Vibro-acoustic analysis is traditionally done in the frequency domain and a broad scale of numerical analysis techniques are available. For low fre-

\footnotetext{
${ }^{*}$ Corresponding author

Email address: Sjoerd.vanOphem@kuleuven.be (S. van Ophem)
} 
quency problems the Finite Element Method (FEM) [1] and the Boundary Element Method (BEM) [2] are the most well known and established techniques. When a vibro-acoustic analysis of the steady state behavior at a limited amount of frequencies is performed, these techniques can achieve very accurate results. One of the main issues of these methods is the need for high computational resources, because of the amount of elements needed per wavelength to keep a low interpolation and pollution error. This results in FEM models typically consisting of a few million Degrees Of Freedom (DOFs). This computational burden can be alleviated with Model Order Reduction (MOR), which can achieve models that are still accurate in the desired frequency range, but with only a fraction of the DOFs. These techniques can be applied to these systems in a relatively straightforward way when only the steady-state frequency response is considered, since the possible loss of stability does not affect the steady-state.

For a transient analysis, MOR needs to be done carefully to assure that the critical or asymptotic stability of the system is preserved. The definition of critical stability states that the system is stable when the generalized eigenvalues of the system have a negative real part [3]. Asymptotic stability is a more restrictive measure, that states that the real part of the generalized eigenvalues should be smaller than zero and thus excludes undamped resonances and rigid-body modes at $0 \mathrm{~Hz}$. Preservation of the stability means that the full system is assumed to be critically stable and that the Reduced Order Model (ROM) is required to have the same stability properties as the original model. The most commonly used MOR techniques are Truncated Balanced Realization (TBR) and Krylov subspace reduction. The TBR scheme is known to give stable ROMs [4], while the ROMs produced by the Krylov subspace projection are not guaranteed to be stable, as has been shown by [5]. Unfortunately, the calculation complexity of the TBR scheme is $O\left(n^{3}\right)$ [6], which is too high for an FE model. The Krylov subspace method, in combination with an Arnoldi scheme and an LU factorization procedure, is more economic for FE models, namely $O\left(k^{2} n\right)$, in which $k$ is the dimension of the reduced order model. Recently, the so called Second Order Arnoldi (SOAR) procedure has been developed for the reduction of second order systems $[7,8]$, having the inherent advantage that the second order structure of the system matrices is preserved. This method is a special case of the structure-preserving Krylov subspace model reduction schemes, described by $\mathrm{Li}$ and Bai [9]. While the SOAR procedure was originally described for moment matching at a single expansion point, the method can also be extended to a rational Krylov interpolation scheme, thus by using multiple expansion points. Wyatt [10] has shown how to extend these methods so that an $H_{2^{-}}$ optimal solution will be obtained, by exploiting an Iterative Rational Krylov 
Arnoldi (IRKA) algorithm, a technique first introduced by Gugercin et al. [11].

For interior vibro-acoustic systems that are modeled with the FEM, the derivation of a stable reduced order model scheme by using a one-side projection has been shown by van de Walle et al. [12]. They show two possible ways of preserving the stability: Firstly, a split basis method, in which the projection matrices are calculated separately for the structural and acoustic domain and coupled afterwards. Secondly, a modified formulation of the resulting differential equations, termed the modified Everstine formulation, for which the authors prove that it preserves the definiteness properties of the system under reduction. In this paper this framework will be extended, so that it can also account for the exterior vibro-acoustic case. This means that the numerical model has to include a boundary that acts as if the Sommerfeld radiation condition is applied [13]. In contrast to the BEM, the FEM does not conform to this condition by default, so it has to be introduced by special means. Several numerical techniques are available to implement this boundary condition. The most well known techniques are the Perfectly Matched Layer (PML) [14], the absorbing boundary condition (ABC) [15], the Dirichlet to Neumann map [13] and Infinite Elements (IE) [16, 17, 18, 19]. The PML is widely used for vibro-acoustics, but mainly in the frequency domain. Time-domain formulations exist [20], but a stable PML interface is still an active research topic, and is therefore not chosen for this work. Since this article focuses on a time domain model, a DtN or an infinite elements approach seem the most suitable, since both approaches have formulations that are derived in the time domain. Here it has to be noted that the DtN map leads to non-sparse system matrices due to its non-local nature, whereas the addition of infinite elements results in sparse system matrices, which is an advantage for the efficiency of Krylov methods. In combination with the notion that infinite elements can be straightforwardly implemented into an FE framework by augmentation of the system matrices, infinite elements are chosen for this work.

Several implementations of the infinite elements exist. The main distinctions are made between the used shape and weighting functions. The earliest implementation of infinite elements uses the same weighting functions and shape functions in the weak formulation of the problem, similar to finite elements, and is usually referred to as the unconjugated IE. This description leads to symmetric, sparse, but frequency dependent system matrices [21]. Astley et al. suggest to change the weighting functions to be the conjugates of the shape functions, leading to the conjugated Astley-Leis formulation $[16,22]$. The consequence is the cancellation of the exponential terms inside the integrals, giving non-symmetric, but frequency independent system 
matrices, which are suitable for a transient system simulation. As has been indicated by Astley et al., the elements approximate the Sommerfeld condition by applying a multipole expansion of a finite order, which determines the accuracy of the elements. It is known that for high order elements the system matrices become ill-conditioned when Lagrangian shape functions are being used, but is has been reported by Dreyer and Von Estorff [23] that these problems can be avoided by using Jacobi polynomials as shape functions. For more details about these shape functions and the different available formulations of IEs, see $[24,25]$. In this paper, the conjugated infinite elements in the Astley-Leis formulation will be used with standard Lagrangian shape functions, because these are most widely used.

The main contribution of this paper is as follows: A procedure for the stability preserving time domain reduction of coupled vibro-acoustic exterior problems using conjugated infinite elements is derived. This is achieved by two approaches that are both based on a one-sided projection method. Proofs are given of the stability preservation for both methods. An additional contribution is the derivation of a stable explicit time domain integration scheme for the reduced order model: Because of a singularity due to the addition of infinite elements, the inversion of the mass matrix is not always possible. A method is thus proposed based on a singular value decomposition, which is also shown to be stability preserving. The layout of the paper is as follows: In Sec. 2 the formulation of the exterior vibro-acoustic problem is given. In Sec. 3 the procedures for the two stability preserving MOR procedures are explained. The stable time integration scheme is derived in Sec. 4, and finally, in Sec. 5 the method is verified with two numerical models, a simple model of a radiating plate and a more involved model of a loudspeaker, where both the frequency and time domain performance are assessed.

\section{Modeling of coupled, unbounded vibro-acoustic systems in the time domain}

In the following section the problem setting used for exterior vibro-acoustic simulations will be described in detail. In Sec. 2.1 the time domain system formulation is given and in Sec. 2.2 the properties of the infinite elements, used for simulating the acoustic pressure field outside the FEM mesh, are given.

\subsection{System formulation in the time domain}

The fully coupled vibro-acoustic system is considered in an FE/IE formulation. The linear system of equations describing the problem can be written in the time domain, as follows: 


$$
\begin{aligned}
M \ddot{x}(t)+C \dot{x}(t)+K x(t) & =F(t), \\
y(t) & =L x(t),
\end{aligned}
$$

in which $M \in \mathbb{R}^{n \times n}$ is the mass matrix, $C \in \mathbb{R}^{n \times n}$ is the damping matrix, $K \in \mathbb{R}^{n \times n}$ is the stiffness matrix and $F(t) \in \mathbb{R}^{n}$ gives the external excitation. Besides the composition of the mesh, the structure of these matrices is dependent on the definition of the state vector $x \in \mathbb{R}^{n}$ and on the domains included in the system. The second line in Eq. (1) describes the resulting output $y(t) \in \mathbb{R}^{q}$, due to the excitation, as function of output matrix $L \in \mathbb{R}^{q \times n}$, and the state vector. In the presented case, there are two physical domains: the structural and acoustic domain. The structural domain is fully described by finite elements, whereas the acoustic domain is subdivided into a finite element domain and an infinite element domain; thus resulting in three numerical domains. The system matrices corresponding to the different physical domains are indicated by a subscript, i.e. $M_{s}$ is the structural mass matrix, $M_{a}$ is the acoustic mass matrix, etc. A coupling between the physical domains is described with a coupling matrix, which is indicated with the subscript $c$.

The structural system matrices and acoustic system matrices do not overlap in the final system matrix assembly, because they describe different DOFs. This is not the case for the system matrices of the acoustic finite elements and the acoustic infinite elements; the outermost DOFs of the FE mesh are shared with the innermost DOFs of the IE mesh, thus the resulting system matrices for the acoustic part of the FE model are summed. In the rest of the paper these shared DOFs are considered to belong to the FE mesh, mainly for ease of notation. For example, the resulting stiffness matrix for the FE part of the acoustic model is

$$
K_{a, 11}=K_{a, F E}+K_{a, I E, \text { inner }},
$$

in which $K_{a, I E, \text { inner }}$ is the part of the stiffness matrix which describes the innermost nodes of the IE mesh, see Fig. 1. The coupling matrices between $K_{a, 11} \in \mathbb{R}^{n_{a, 11} \times n_{a, 11}}$ and the rest of the IE mesh will be named $K_{a, 12} \in \mathbb{R}^{n_{a, 11} \times n_{a, 22}}$ and $K_{a, 21} \in \mathbb{R}^{n_{a, 22} \times n_{a, 11}}$ and the stiffness matrix of the rest of the infinite elements will be $K_{a, I E, \text { outer }}=K_{a, 22} \in \mathbb{R}^{n_{a, 22} \times n_{a, 22}}$. This results in the following acoustic stiffness matrix: 


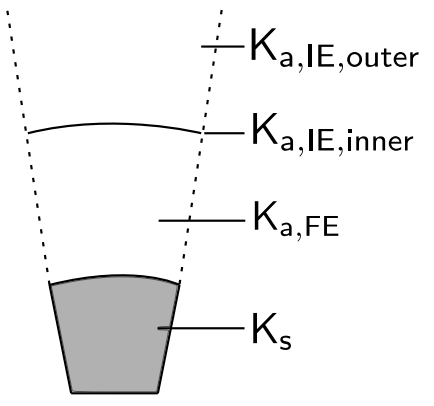

Figure 1: Subdivision of the domains

$$
\begin{aligned}
& K_{a, t o t}=\left[\begin{array}{cc}
K_{a, F E}+K_{I E, \text { inner }} & K_{a, 12} \\
K_{a, 21} & K_{a, I E, \text { outer }}
\end{array}\right] \\
& =\left[\begin{array}{ll}
K_{a, 11} & K_{a, 12} \\
K_{a, 21} & K_{a, 22}
\end{array}\right] \text {. }
\end{aligned}
$$

The mass and damping matrices will be named in the same manner as the stiffness matrix. A possible way for assembly of the system matrices is as follows:

$$
\begin{aligned}
M_{e u l}= & {\left[\begin{array}{ccc}
M_{s} & 0 & 0 \\
-\rho K_{c}^{T} & M_{a, 11} & M_{a, 12} \\
0 & M_{a, 21} & M_{a, 22}
\end{array}\right], C_{\text {eul }}=\left[\begin{array}{ccc}
C_{s} & 0 & 0 \\
0 & C_{a, 11} & C_{a, 12} \\
0 & C_{a, 21} & C_{a, 22}
\end{array}\right], } \\
K_{\text {eul }}= & {\left[\begin{array}{ccc}
K_{s} & K_{c} & 0 \\
0 & K_{a, 11} & K_{a, 12} \\
0 & K_{a, 21} & K_{a, 22}
\end{array}\right], x_{\text {eul }}=\left[\begin{array}{c}
u \\
p_{F E} \\
p_{I E}
\end{array}\right] . }
\end{aligned}
$$

This form is well known as the Eulerian formulation, as indicated by the subscript (.) eul, which solves for the state variables $u, p_{F E}$ and $p_{I E} . u \in \mathbb{R}^{n_{s}}$ is a vector containing the structural displacements and rotations at the structural nodes, $p_{F E} \in \mathbb{R}^{n_{a, 11}}$ is a vector containing the acoustic pressures at the acoustic FE nodes and $p_{I E} \in \mathbb{R}^{n_{a, 22}}$ is a vector containing acoustic pressures at the IE nodes. The last term has a special form in the time domain, as is shown in Sec. 2.2. Furthermore, the matrices belonging to the finite element mesh are assumed to have their usual definiteness properties: $M_{s}, C_{s}$ and $M_{a, F E}$ are positive definite, $K_{s}, K_{a, F E}$ and $C_{a}$ are positive semidefinite, and all of the FE matrices are symmetric [1]. For the infinite elements the matrix properties are somewhat different, as described in the following sec- 
tion. Throughout the whole paper the excitation is assumed to be in the FE domain.

The stated formulations will be used throughout the paper and can be used with the Eulerian formulation, as well as the (modified) Everstine formulation, of which the details are described in Sec. 3.3.

\subsection{State variables for the infinite elements}

As already indicated in Sec. 2.1, infinite elements will be added to account for the Sommerfeld radiation condition needed for unbounded problems. The conjugated Astley-Leis infinite elements are chosen as the preferred implementation. The solution of the states in the IE domain is given by the inverse Fourier transform of the solution in the frequency domain. When a system formulation is used in which the pressure amplitudes in the acoustic part of the FE mesh are calculated, this solution is assumed to be of the following form, for a radial frequency $\omega=2 \pi f[17]$ :

$$
\bar{p}_{l}(\omega)=\bar{q}_{l}(\omega) e^{-i(\omega / c) d_{l}},
$$

in which $\bar{q}_{l}(\omega)$ is the solution following from inversion of the system matrices and multiplication with the force vector, $d_{l}$ is the distance between node $l$ and the innermost node of the IE mesh and $c$ is the speed of sound. The time domain equivalent of this solution can be found by the using the time shifting property of the inverse Fourier transform:

$$
q_{l}(t)=\mathcal{F}^{-1}\left\{\bar{q}_{l}(\omega)\right\}=\mathcal{F}^{-1}\left\{\bar{p}_{l}(\omega) e^{i(\omega / c) d_{l}}\right\}=p_{l}\left(t+d_{l} / c\right) .
$$

This is simply the time advanced pressure on this position, in which the delay time is the distance $d_{l}$ between the node and the most inner node of the infinite element domain, divided by the sound propagation speed $c$. This observation will be used in Sec. 3.3 to derive a time stable formulation.

\subsection{Stability considerations for different formulations}

There are two possible formulations for conjugated infinite elements: mapped and spheroidal elements, as illustrated in Fig. 2. The stability of the infinite elements is dependent on both the chosen element formulation and the geometry of the interface layer between the finite and infinite elements. As has been indicated in [26] the required condition for stability of the infinite elements is the semi-positive definiteness of the mass matrix. This was also shown numerically by Astley and Hamilton [18], where the authors concluded that for mapped elements only stable results are acquired when the infinite elements are locally orthogonal to the interface, which happens when the interface is a sphere. The authors show that in this specific case the mass 

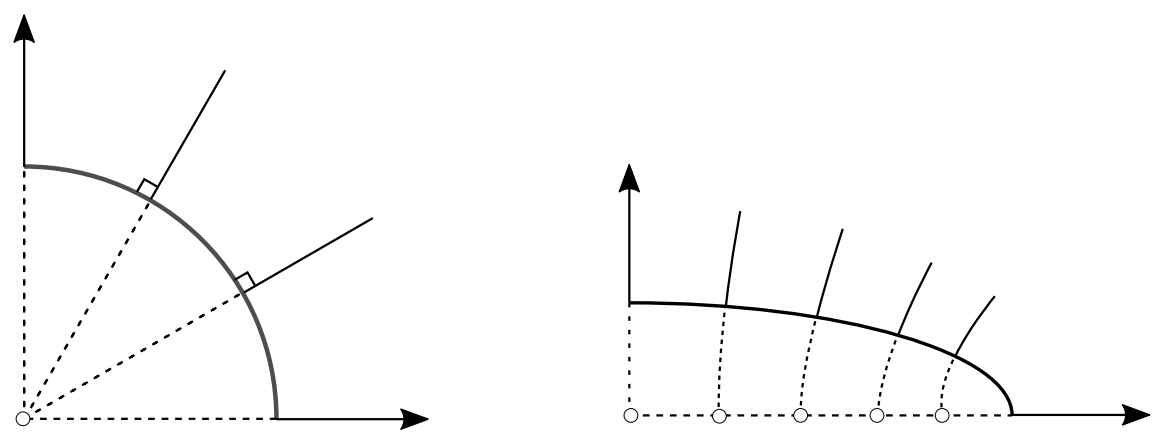

Figure 2: Mapped element shape (left) and spheroidal elements (right)

matrix for the infinite elements is zero, and that if the mass matrix is not exactly zero, due to small inconsistencies in the geometry, that the system becomes unstable, because the mass matrix loses its semi-positive definiteness properties. On the other hand, the authors show that a spheroidal formulation will give stable results for any spheroidal interface layer.

For the implementation of the infinite elements in the time domain, the possible inclusion of the singularity in the mass matrix has to be taken into account, as discussed in more detail in Sec. 4. The accuracy of the infinite elements is dependent on the order of the elements and the aspect ratio of the interface. A higher aspect ratio needs a higher order infinite element layer to approximate the solution with sufficient accuracy. This might lead to numerical problems, since the conjugated Astley-Leis infinite elements are known to get ill-conditioned for orders higher than 10. For a detailed comparison for the different formulations, the reader is referred to Astley and Hamilton [21]. Using Jacobi polynomials may lead to more accurate results for higher order shape functions, as has been suggested by Dreyer [23], but this is outside the scope of this paper.

\section{Stability preserving model order reduction for exterior time do- main vibro-acoustics}

This section describes the required properties of the system to arrive at a reduced order model that preserves stability of the original full system. To assess the stability, the second order system will be converted to an equivalent first order system in Sec. 3.1. Consequently, the stability demands of this possibly singular, non-symmetric descriptor system will be given in Sec. 3.2 and the stability under a congruence transform will assessed for several system formulations in Sec. 3.3. Finally, in Sec. 3.4 two different MOR procedures will be described that both preserve the stability of the full system. 


\subsection{Transformation of the second order system to descriptor form}

The second order system will be transformed to a first-order system, because the methods for determining the stability of second order systems are in general less developed and more difficult to assess than for first-order systems. Eq. (1) can be rewritten as a set of first order differential equations, by rewriting the equations in descriptor form. By reformulating the external excitation as the matrix-vector product $F(t)=G u(t)$, in which $G \in \mathbb{R}^{n}$ is the input vector, and $u(t)$ is the input signal, a possible (non-unique) formulation is

$$
\begin{aligned}
\underbrace{\left[\begin{array}{cc}
I & 0 \\
0 & M
\end{array}\right]}_{\mathrm{E}} \underbrace{\left[\begin{array}{c}
\dot{x}(t) \\
\ddot{x}(t)
\end{array}\right]}_{\dot{x}}=\underbrace{\left[\begin{array}{cc}
0 & I \\
-K & -C
\end{array}\right]}_{\mathrm{A}} \underbrace{\left[\begin{array}{c}
x(t) \\
\dot{x}(t)
\end{array}\right]}_{\mathrm{x}}+\underbrace{\left[\begin{array}{c}
0 \\
G
\end{array}\right]}_{\mathrm{B}} u(t), \\
y(t)=\underbrace{\left[\begin{array}{ll}
L & 0
\end{array}\right]}_{\mathrm{L}} \underbrace{\left[\begin{array}{c}
x(t) \\
\dot{x}(t)
\end{array}\right]}_{\mathrm{x}} .
\end{aligned}
$$

This system description can be converted to a state space formulation by inversion of the mass matrix. This can only be done when the mass matrix is non-singular:

$$
\begin{aligned}
{\left[\begin{array}{l}
\dot{x}(t) \\
\ddot{x}(t)
\end{array}\right] } & =\left[\begin{array}{cc}
0 & I \\
-M^{-1} K & -M^{-1} C
\end{array}\right]\left[\begin{array}{l}
x(t) \\
\dot{x}(t)
\end{array}\right]+\left[\begin{array}{c}
0 \\
M^{-1} G
\end{array}\right] u(t), \\
y(t) & =\left[\begin{array}{ll}
L & 0
\end{array}\right]\left[\begin{array}{l}
x(t) \\
\dot{x}(t)
\end{array}\right] .
\end{aligned}
$$

The inclusion of infinite elements may lead to a singular mass matrix, making a straightforward inversion of the mass matrix impossible. This may lead to problems for efficient time integration, as is discussed in more detail in Sec. 4.

\subsection{Stability of possibly singular descriptor systems, resulting from the in-} clusion of infinite elements

The system is required to be stable in the time domain, which is the case when the system matrices conform to certain definiteness properties. As stated in the introduction, the system is called critically stable when the real part of the eigenvalues resulting from the generalized eigenvalue problem $(E, A)$ in Eq. (7) is smaller than, or equal to zero [3] and the system is called exponentially stable when all the real parts of the eigenvalues are smaller 
than zero. In the rest of this paper the notion of critical stability will be used when stability is discussed, unless stated otherwise. The stability should be preserved when the model of the system is reduced and when the model is discretized in the time domain. The stiffness, damping and mass matrices resulting from the infinite elements can be non-symmetric [16]. Therefore, a generic description of positive (semi-)definiteness is used, that allows for non-symmetry. Suppose that a non-symmetric matrix $A \in \mathbb{R}^{n \times n}$ is analyzed. The matrix is defined positive definite if

$$
\mathfrak{R e}\left\{x^{T} A x\right\}>0, \forall x \in \mathbb{R}^{n} .
$$

This will be notated as follows: $A>0$. For a negative (semi-) definite matrix a similar description will be used $(A<0)$.

Lemma 1. A descriptor system is stable if $E \geq 0$ and $A \leq 0$. Note, this is different from Theorem 1 in Ref. [12], since in this work the matrix $E$ is only semi-positive definite.

\section{Proof. See Appendix A.}

It is now shown that, under the assumption of the matrix properties that are described in Secs. 2.1 and 2.3 that the full system conforms to the property that is described in Lemma 1.

As has been described in Sec. 2.3, the IEs lead to similar definiteness properties as the coupled finite element matrices without the IEs, except for the mass matrix, which is now possibly only positive semi-definite, a different first order formulation is used:

$$
\underbrace{\left[\begin{array}{cc}
K^{T} & 0 \\
0 & M
\end{array}\right]}_{\mathrm{E}}\left[\begin{array}{l}
\dot{x} \\
\ddot{x}
\end{array}\right]=\underbrace{\left[\begin{array}{cc}
0 & K^{T} \\
-K & -C
\end{array}\right]}_{\mathrm{A}}\left[\begin{array}{l}
x \\
\dot{x}
\end{array}\right]+\left[\begin{array}{l}
0 \\
G
\end{array}\right] u .
$$

Note that the substitution of $I$ for $K^{T}$ still leads to the same second order differential equation as before. The exact form of $K, C$ and $M$ is dependent on the chosen formulation.

Lemma 2. The system in Eq. (12) is stable when $M \geq 0, K \geq 0$ and $C \geq 0$.

Proof. See Appendix B. 


\subsection{Congruence transform on coupled system matrices and its stability for different system formulations}

The MOR schemes that are described in this paper calculate the ROM by using a projection basis $V \in \mathbb{C}^{n \times k}$, in which $k$ is the size of the reduced system. This is done by a congruence transform of the following form

$$
A_{r}=V^{T} A V,
$$

in which $A$ is the original matrix, and $A_{r}$ is the reduced order matrix. It has been shown in [12], that this transformation preserves the definiteness properties of the full matrix $A$. If the fully assembled stiffness, damping and mass matrices do not exhibit the properties of the form specified in Lemma 2, then the resulting reduced order system is not guaranteed to be stable. The coupled system can be written in different formats, leading to different definiteness properties of the fully assembled system matrices. In the following section two formulations are compared.

The Eulerian model already has been discussed in Sec. 2.1. The main advantages of this formulation are that in the acoustic domain the pressure is obtained directly as a field variable and that most FE solvers use this formulation. With the assumptions made in Sec. 3.2, an analysis of the stability under a congruence transform can be done, by looking at the definiteness properties of the resulting system matrices. As has already been shown in [12], the coupled case without infinite elements gives a potentially unstable system. This is also the case for the coupled system with infinite elements, as is shown here:

$$
\begin{aligned}
\mathfrak{R e}\left\{x^{T} K_{\text {eul }} x\right\} & =\left[\begin{array}{lll}
x_{1}^{T} & x_{2}^{T} & x_{3}^{T}
\end{array}\right]\left[\begin{array}{ccc}
K_{s} & K_{c} & 0 \\
0 & K_{a, 11} & K_{a, 12} \\
0 & K_{a, 21} & K_{a, 22}
\end{array}\right]\left[\begin{array}{l}
x_{1} \\
x_{2} \\
x_{3}
\end{array}\right] \\
& =x_{1}^{T} K_{s} x_{1}+x_{2}^{T} K_{c} x_{1}+\left[\begin{array}{ll}
x_{2}^{T} & x_{3}^{T}
\end{array}\right]\left[\begin{array}{ll}
K_{a, 11} & K_{a, 12} \\
K_{a, 21} & K_{a, 22}
\end{array}\right]\left[\begin{array}{l}
x_{2} \\
x_{3}
\end{array}\right](.14)
\end{aligned}
$$

Since the assembled stiffness matrix of the acoustic part is by assumption semi-positive definite, this means that the stability is determined by the vibro-acoustic coupling term $x_{2}^{T} K_{c} x_{1}$. The value of this term is not known and therefore, stability under a congruence transform cannot be guaranteed.

As has been shown in [12], for the interior vibro-acoustics case, the stability under model order reduction can be guaranteed by using a modified $u-\phi$ formulation. This formulation calculates the fluid velocity potentials $\phi$ in the acoustic nodes, instead of the pressures, see Everstine [27]. The 
velocity potential and the pressure are related by $p=-\rho \dot{\phi}$. The formulation used here is called modified, because some of the matrices have a sign change, as compared to the original Everstine formulation. In Sec. 2.2 it has been shown that the field variables resulting from the infinite elements in the time domain simply are time advanced pressure terms, thus making them suitable for the $u-\phi$ formulation, by using $q_{l}(t)=p_{l}\left(t+d_{l} / c\right)=-\rho \dot{\phi}_{l}\left(t+d_{l} / c\right)$. The modified formulation is as follows, where the subscript (.) eve is added to indicate that the matrices are written in the Everstine formulation:

$$
\begin{gathered}
M_{\text {eve }}=\left[\begin{array}{ccc}
M_{s} & 0 & 0 \\
0 & \rho M_{a, 11} & \rho M_{a, 12} \\
0 & \rho M_{a, 21} & \rho M_{a, 22}
\end{array}\right], C_{\text {eve }}=\left[\begin{array}{ccc}
C_{s} & -\rho K_{c} & 0 \\
\rho K_{c}^{T} & \rho C_{a, 11} & \rho C_{a, 12} \\
0 & \rho C_{a, 21} & \rho C_{a, 22}
\end{array}\right], \\
K_{\text {eve }}=\left[\begin{array}{ccc}
K_{s} & 0 & 0 \\
0 & \rho K_{a, 11} & \rho K_{a, 12} \\
0 & \rho K_{a, 21} & \rho K_{a, 22}
\end{array}\right], x_{\text {eve }}=\left[\begin{array}{c}
u \\
\phi_{F E} \\
\phi_{I E}
\end{array}\right], F_{\text {eve }}=\left[\begin{array}{c}
F_{s} \\
-F_{\phi, F E} \\
0
\end{array}\right] .
\end{gathered}
$$

As shown in [12] to preserve stability during model order reduction of vibro-acoustic systems for interior problems, it is required to adjust the Everstine formulation, by changing the sign of the acoustic matrices. It is shown here that for the exterior acoustics case with infinite elements the same conclusion can be drawn. The stability will be preserved, because the coupling matrices vanish from the stiffness and mass matrix expressions and give the following properties for the damping matrix:

$$
\begin{aligned}
\mathfrak{R e}\left\{x^{T} C_{\text {eve }} x\right\} & =\left[\begin{array}{lll}
x_{1}^{T} & x_{2}^{T} & x_{3}^{T}
\end{array}\right]\left[\begin{array}{ccc}
C_{s} & -\rho K_{c} & 0 \\
\rho K_{c}^{T} & \rho C_{a, 11} & \rho C_{a, 12} \\
0 & \rho C_{a, 21} & \rho C_{a, 22}
\end{array}\right]\left[\begin{array}{l}
x_{1} \\
x_{2} \\
x_{3}
\end{array}\right] \\
& =x_{1}^{T} C_{s} x_{1}+\rho\left[\begin{array}{ll}
x_{2}^{T} & x_{3}^{T}
\end{array}\right]\left[\begin{array}{ll}
C_{a, 11} & C_{a, 12} \\
C_{a, 21} & C_{a, 22}
\end{array}\right]\left[\begin{array}{l}
x_{2} \\
x_{3}
\end{array}\right] \geq 0 .
\end{aligned}
$$

This means that also for the exterior vibro-acoustic model the modified Everstine formulation is suitable for model order reduction with a congruence transform.

\subsection{Model order reduction procedure}

Two possible reduction strategies will be discussed, namely (i) reducing the calculation domains separately and (ii) reducing the whole system simultaneously. Both strategies start by calculating the reduced order basis 
$V \in \mathbb{R}^{n \times k}$, used as transformation matrix of the congruence transform. In the numerical examples in Sec. 5 an example is given of how to calculate $V$ with aid of the Second Order Arnoldi (SOAR) algorithm, which is a numerical procedure specifically designed for second order systems, see [8] for details. The SOAR algorithm directly projects the $M, C$ and $K$ matrices to a reduced form. The congruence transform results in the following reduced equations:

$$
\begin{aligned}
M_{r} \ddot{x}_{r}(t)+C_{r} \dot{x}_{r}(t)+K_{r} x_{r}(t) & =B_{r} u(t), \\
y(t) & =L_{r}(t),
\end{aligned}
$$

with

$$
\begin{aligned}
M_{r} & =V^{T} M V, K_{r}=V^{T} K V, \\
C_{r} & =V^{T} C V, B_{r}=V^{T} B, \\
L_{r} & =L V, x(t) \approx V x_{r} .
\end{aligned}
$$

\subsubsection{Splitting the calculation domains}

The calculation of $V$, is based on the full system, and has the following structure:

$$
V=\left[\begin{array}{c}
V_{s} \\
V_{a, 11} \\
V_{a, 22}
\end{array}\right] \in \mathbb{R}^{n \times k},
$$

with $V_{s} \in \mathbb{R}^{n_{s} \times k}, V_{a, 11} \in \mathbb{R}^{n_{a, 11} \times k}, V_{a, 22} \in \mathbb{R}^{n_{a, 22} \times k}$. One of the options for stable model order reduction is to split $V$ into the 3 calculation domains as follows:

$$
V_{\text {tot }}=\left[\begin{array}{ccc}
\operatorname{orth}\left(V_{s}\right) & 0 & 0 \\
0 & \operatorname{orth}\left(V_{a, 11}\right) & 0 \\
0 & 0 & \operatorname{orth}\left(V_{a, 22}\right)
\end{array}\right] .
$$

Orthonormalization is applied on the separate matrices, since a split of $V$ does not always lead to submatrices of full rank. This method works for all the aforementioned formulations, so also for the Eulerian formulation, because it leaves the structure of the individual domain matrices intact. An advantage of this method is the ease of application on existing finite element codes, but it also has several disadvantages. First of all, it reduces all the individual domains to approximately the same size, leading to a possible 
discrepancy in accuracy between the computational domains. Furthermore, in general it does not give reduced order models of an optimal size [28].

\subsubsection{Applying MOR on the whole system simultaneously}

An alternative strategy is to apply MOR without splitting the basis. As has been discussed in Sec. 3.3, in this case the system has to be put into a modified $u-\phi$ formulation to arrive at a system that is stable under a single congruence transform. After calculating the reduced order basis $V$, the reduced order system matrices can be calculated. Here care has to be taken, since the reduced order mass matrix will only be calculated with a part of the reduced basis, as will be shown here:

Assume the following definitions:

$$
\begin{aligned}
V_{1} & =\left[\begin{array}{c}
V_{s} \\
V_{a, 11}
\end{array}\right], V_{2}=V_{a, 22}, \\
M_{11} & =\left[\begin{array}{cc}
M_{s} & 0 \\
0 & \rho M_{a, 11}
\end{array}\right] .
\end{aligned}
$$

When a spherical IE boundary is added, the contribution of the IEs to the mass matrix is zero [18], thus $M_{a, 12}, M_{a, 21}$ and $M_{a, 22}$ are zero, and the reduced order matrix will have the following definition:

$$
M_{r}=\left[\begin{array}{ll}
V_{1}^{T} & V_{2}^{T}
\end{array}\right]\left[\begin{array}{cc}
M_{11} & 0 \\
0 & 0
\end{array}\right]\left[\begin{array}{l}
V_{1} \\
V_{2}
\end{array}\right]=V_{1}^{T} M_{11} V_{1} .
$$

The sub-matrix $V_{1}$ is not assumed to be of full rank and therefore the reduced order mass matrix is possibly singular, since the columns may not be linearly independent. It is thus not guaranteed that inversion of the mass matrix is possible. It can be checked if this is the case by calculating the rank of the sub matrix $V_{1}$. If the rank of this matrix is lower than the amount of columns, then the reduced order mass matrix will be singular. A solution to this problem is given in Sec. 4.2. Otherwise a mass matrix inversion can be applied, as given in Eq. (9). The advantages of using this MOR method, as compared to the method described in Sec. 3.4.1, are that in general the resulting model size will be smaller for the same accuracy and that the individual sub-domains will be scaled appropriately in the reduced order model. A comparison between the split and non split formulation is given in Table 1. 


\begin{tabular}{cccc} 
& & Split & Non split \\
\hline \multirow{2}{*}{$u-p$} & Stability & Stable & Unstable \\
& Number of DOFs & $\sim 3 n$ & $n$ \\
\hline \multirow{2}{*}{$u-\phi$} & Stability & Stable & Stable \\
& Number of DOFs & $\sim 3 n$ & $n$ \\
\hline
\end{tabular}

Table 1: Comparison of the ROMs resulting from the split and the non plit basis for the $u-p$ and $u-\phi$ formulation.

\section{Efficient time integration of singular descriptor systems}

As already stated in Sec. 2.3, the infinite elements can have a singular mass matrix description. In this case an inversion as shown in Eq. (9) to arrive at a state space model is not possible. This means that time integration has to be performed on the descriptor system in Eq. (7), which has to be done with implicit time integration schemes, such as Newmark- $\beta$. It is known that implicit time integration schemes can be computationally demanding, because a matrix inversion has to be performed at every time step. Since the goal of time domain MOR is to reduce the calculation complexity as much as possible, it would be beneficial if an explicit time integration scheme could be used. To do this, the first order system will be transformed from its descriptor form to a state space model, as is described in Secs. 4.1-4.2. Since the described transformation relies on a singular value decomposition, it is computationally demanding. Therefore, in Sec. 4.3 it is shown that this transformation can be performed on the ROMs as well, instead of the full model. This section concludes with the description of the resulting explicit time integration scheme, in Sec. 4.4.

\subsection{Index of the descriptor system}

Consider the following definition for the state vector:

$$
x_{1}=\left[\begin{array}{c}
u \\
\phi_{F E}
\end{array}\right], x_{2}=\phi_{I E}, x_{f u l l}=\left[\begin{array}{l}
x_{1} \\
x_{2}
\end{array}\right] \text {. }
$$

When the IEs are applied on a spherical boundary and thus no additional mass due to the IEs is added to the system matrices, the following descriptor system results from Eq. (7):

$$
\left[\begin{array}{cccc}
I & 0 & 0 & 0 \\
0 & I & 0 & 0 \\
0 & 0 & M_{11} & 0 \\
0 & 0 & 0 & 0
\end{array}\right]\left[\begin{array}{l}
\dot{x}_{1} \\
\dot{x}_{2} \\
\ddot{x}_{1} \\
\ddot{x}_{2}
\end{array}\right]=\left[\begin{array}{cccc}
0 & 0 & I & 0 \\
0 & 0 & 0 & I \\
-K_{11} & -K_{12} & -C_{11} & -C_{12} \\
-K_{21} & -K_{22} & -C_{21} & -C_{22}
\end{array}\right]\left[\begin{array}{l}
x_{1} \\
x_{2} \\
\dot{x}_{1} \\
\dot{x}_{2}
\end{array}\right]+B u,
$$


where $K_{i i}$ and $C_{i i}$ are defined in a similar way as Eq. (24). For a nonsingular $E$-matrix the descriptor system describes a full set of first order ordinary differential equations (ODEs). By defining the full state vector as $x_{\text {full }}=\left[\begin{array}{llll}x_{1} & x_{2} & \dot{x}_{1} & \dot{x}_{2}\end{array}\right]^{T}$, the resulting descriptor system now describes a full set of ODEs of the form $E \dot{x}_{\text {full }}(t)=A x_{\text {full }}(t)$. However, with a singular matrix $E$, also a set of algebraic equations are introduced; equations that only depend on $x_{\text {full }}$ and not on its derivatives. The combined set of equations is called a differential algebraic equation (DAE). The procedure for solving these DAEs is dependent on the differentiation index, which is defined as the amount of derivatives required to obtain a set of ODEs from the $\mathrm{DAE}^{1}$ [30]. In the case of Eq. (27), to convert the system to a set of ordinary differential equations, the fourth line of the set of equations has to be differentiated once, which gives

$$
\left[\begin{array}{cccc}
I & 0 & 0 & 0 \\
0 & I & 0 & 0 \\
0 & 0 & M_{11} & 0 \\
0 & 0 & C_{21} & C_{22}
\end{array}\right]\left[\begin{array}{l}
\dot{x}_{1} \\
\dot{x}_{2} \\
\ddot{x}_{1} \\
\ddot{x}_{2}
\end{array}\right]=\left[\begin{array}{cccc}
0 & 0 & I & 0 \\
0 & 0 & 0 & I \\
-K_{11} & -K_{12} & -C_{11} & -C_{12} \\
0 & 0 & -K_{21} & -K_{22}
\end{array}\right]\left[\begin{array}{l}
x_{1} \\
x_{2} \\
\dot{x}_{1} \\
\dot{x}_{2}
\end{array}\right]+B u
$$

This means that the differential algebraic equation is of index-1. One might ask if the derived expression can be used directly in a time domain integration scheme. Since the new system matrices are a combination of different subsets of the old system matrices, positive definiteness of the resulting system cannot be guaranteed and therefore stability cannot be guaranteed under a single congruence transform. In the following sub-section a method is shown which converts the system to state space form without the inversion of the mass matrix and which preserves stability properties. This method was first proposed by Kumar [30].

\subsection{Decomposition of the E-matrix}

Suppose that a descriptor system of index-1 with a matrix $E \in \mathbb{R}^{n \times n}$ is given, thus $E$ is singular. The descriptor system has the following form (leaving away the external excitation matrix for brevity of notation):

\footnotetext{
${ }^{1}$ Depending on the index, the response of the system has to be adjusted to take into account dynamic effects of the algebraic equations. It has been shown in [29] that for an index-1 system the algebraic constraints add a constant term that is dependent on the external excitation of the algebraic equations. For the systems described in this paper no external excitation is applied to the algebraic equations, since there is no external excitation within the IE domain, thus the constant term is zero and the resulting response does not require an adjustment.
} 


$$
E \dot{x}=A x .
$$

A decomposition of the matrix $E$ can be done:

$$
E=X Y^{T}
$$

If the matrices $X$ and $Y$ have full column rank, then the decomposition is called a full rank decomposition of $E$. When the matrix $E$ will be of $\operatorname{rank}(E)=r$ with $r \leq n$, then $X, Y \in \mathbb{R}^{n \times r}$ and $\operatorname{rank}(X)=\operatorname{rank}(Y)=r$. A possible way to achieve this decomposition is given in Algorithm 1.

Eq. (29) can be rewritten as follows:

$$
X Y^{T} \dot{x}(t)=A x(t) .
$$

If $A$ is invertible, which is the case when the full system is asymptotically stable, both sides of the equation can be premultiplied with $Y^{T} A^{-1}$, which gives

$$
Y^{T} A^{-1} X Y^{T} \dot{x}(t)=Y^{T} x(t) .
$$

Define the new state variable $z(t)$ as follows:

$$
z(t)=Y^{T} x(t)
$$

and also

$$
\tilde{E}=Y^{T} A^{-1} X
$$

Thus the equation can be rewritten to

$$
\tilde{E} \dot{z}(t)=z(t) .
$$

In [30] a proof is given which shows that the eigenvalues of the derived state space system of index-1 are the same as the eigenvalues derived from the generalized eigenvalue problem; in other words, the finite eigenvalues of $(E, A)$ and $(\tilde{E}, I)$ are the same and therefore the transform does not affect the stability of the system ${ }^{2}$.

This leads to the following state space system

\footnotetext{
${ }^{2}$ Note that the algorithm presented above also could be used for systems with illconditioned system matrices. By setting a tolerance in the algorithm on the singular values, every singular value below this value will be considered zero, and therefore will lead to a rank reduction.
} 


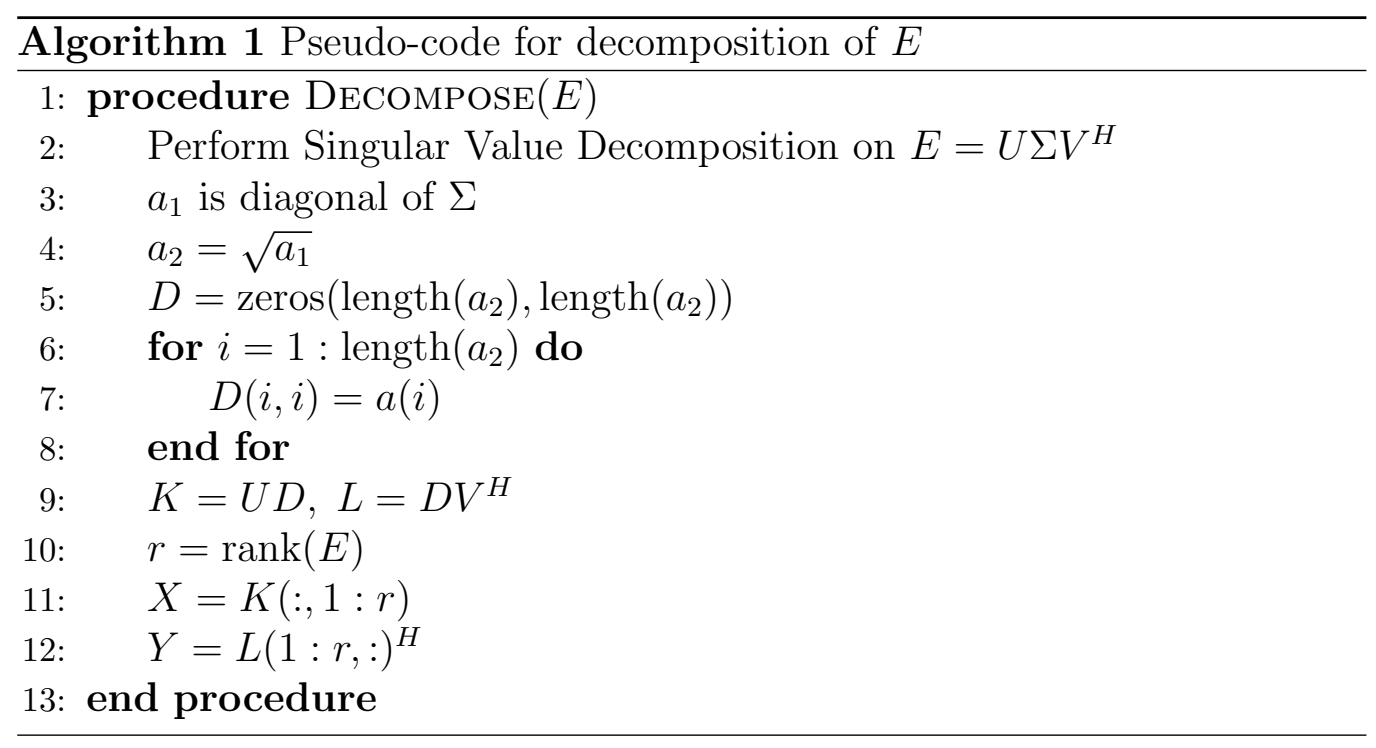

$$
\begin{aligned}
\dot{z}(t) & =\tilde{A} z(t)+\tilde{B} u(t) \\
y(t) & =\tilde{C} z(t)
\end{aligned}
$$

with the following state matrices [30]:

$$
\begin{aligned}
\tilde{A} & =\left(Y^{T} A^{-1} X\right)^{-1}, \\
\tilde{B} & =\tilde{A} Y^{T} A^{-1} B \\
\tilde{C} & =C A^{-1} X \tilde{A}, \\
\tilde{D} & =D-C^{T} A^{-1} B+\tilde{C} Y^{T} A^{-1} B .
\end{aligned}
$$

Since the eigenvalues of the system are not changed, but the singular matrix $E$ is successfully removed, the matrices can be used for time integration.

\subsection{Preservation of the index under different MOR schemes}

The transformation method used to transform from a singular descriptor system to a state space system, as described in Sec. 4.2, will be computationally demanding if it is performed on the full system matrices. In this section it is shown that this is not necessary and that the transformation can be applied on the ROMs instead, both for the method in which the reduced base is split for the different calculation domains and where the base remains intact. 


\subsubsection{Splitting the calculation domains}

When the MOR strategy from Sec. 3.4.1 is applied on the system matrices, the structure of the system will be preserved, thus the mass matrix is again singular:

$$
M_{r}=V_{t o t}^{T}\left[\begin{array}{ccc}
M_{s} & 0 & 0 \\
-\rho K_{c}^{T} & M_{a, 11} & 0 \\
0 & 0 & 0
\end{array}\right] V_{t o t}=\left[\begin{array}{ccc}
M_{s, r} & 0 & 0 \\
-\rho K_{c, r}^{T} & M_{a_{11}, r} & 0 \\
0 & 0 & 0
\end{array}\right] .
$$

This means that the transformation in Sec. 4 can be applied on the reduced system without problems, since the reduced system is again a DAE of index-1.

\subsubsection{Applying MOR on the whole system simultaneously}

For the MOR strategy described in Sec. 3.4.2 also the transformation can be done on the reduced models. This can be done, because the index-1 descriptor system is still implicitly present in the system matrices. As has been been shown in [12] and discussed in Sec. 3.3, the definiteness properties of the system matrices do not change when a congruence transform is applied on them. Also, as has been explained in Sec. 3.4.2, only part of the projection matrix is used to calculate the reduced mass matrix, and there is no guarantee that this sub-matrix is of full rank. This means that the mass matrix can still be singular after reduction, thus that again a DAE of index-1 results. Even when $M_{r}$ is of full rank, it was found that the resulting matrix is often ill-conditioned, thus that the transformation from descriptor to state space form by performing a full rank matrix decomposition on $E$ is preferred to a straightforward inversion of $M_{r}$.

\subsection{Exponential time integration}

Until now, the equations are only discretized in the spatial domain. To calculate the solution as function of time, a time integration scheme has to be chosen to discretize the system in the time domain. In this paper, an explicit exponential time integration scheme is chosen, with a zero order hold input, since it gives accurate results if the sampling frequency $f_{s}$ is chosen high enough and it is computationally efficient. Moreover, with the enforced structure of the eigenvalues in the previous sections the stability of the integration scheme can be proven, see Appendix C, which is usually the main argument against using explicit schemes. The numerical integration scheme is given by 


$$
\begin{aligned}
x[i+1] & =A_{d} x[i]+B_{d} u[i], \\
y[i] & =C_{d} x[i]+D_{d} u[i],
\end{aligned}
$$

in which

$$
\begin{aligned}
& A_{d}=e^{\tilde{A} T_{s}}, B_{d}=\tilde{A}^{-1}\left(A_{d}-I\right) \tilde{B} \\
& C_{d}=\tilde{C}, D_{d}=\tilde{D}, T_{s}=1 / f_{s} .
\end{aligned}
$$

In this paper, all the examples in which the exponential integrator is used are calculated with the algorithm described in Ref. [31].

\section{Numerical verification}

In the following section the described procedure will be verified with two numerical models. The first numerical model is an academic example, in which the geometry is kept simple on purpose. The geometry comprises a plate and a sphere with acoustic FEs and IEs on the boundary. In this example the $u-p$ and modified $u-\phi$ form and their related stability properties are shown and their accuracy is compared both in the frequency and time domain. Furthermore, the efficient exponential time integration scheme is compared to a Newmark- $\beta$ integration scheme, see Sec. 5.1. The second model describes the vibro-acoustic behavior of a loudspeaker, which is an example of a typical industrial vibro-acoustic problem. This model is included to show that the methodology also works on models with high complexity. Also for this model a comparison is done between the accuracy in the frequency domain and time domain, see Sec. 5.2.

\subsection{Model 1: Plate radiating sound into the free field}

The first example comprises a coupled vibro-acoustic system which consists of a rectangular plate that is excited by a force and radiates sound in the free field. The physical properties of the plate are given in Table 2. For the acoustic domain air at room temperature is used $(c=343 \mathrm{~m} / \mathrm{s}, \rho=$ $\left.1.225 \mathrm{~kg} / \mathrm{m}^{3}\right)$. The plate is modeled with triangular shell elements with linear shape functions and the size of the elements is chosen such that at least 6 elements per wavelength are included at the highest frequency of interest, which is set at $f=500 \mathrm{~Hz}$. The acoustic part of the mesh is modeled with tetrahedral elements containing linear shape functions. The boundary layer between the finite and infinite elements is set at $1 \mathrm{~m}$ from the center of the 

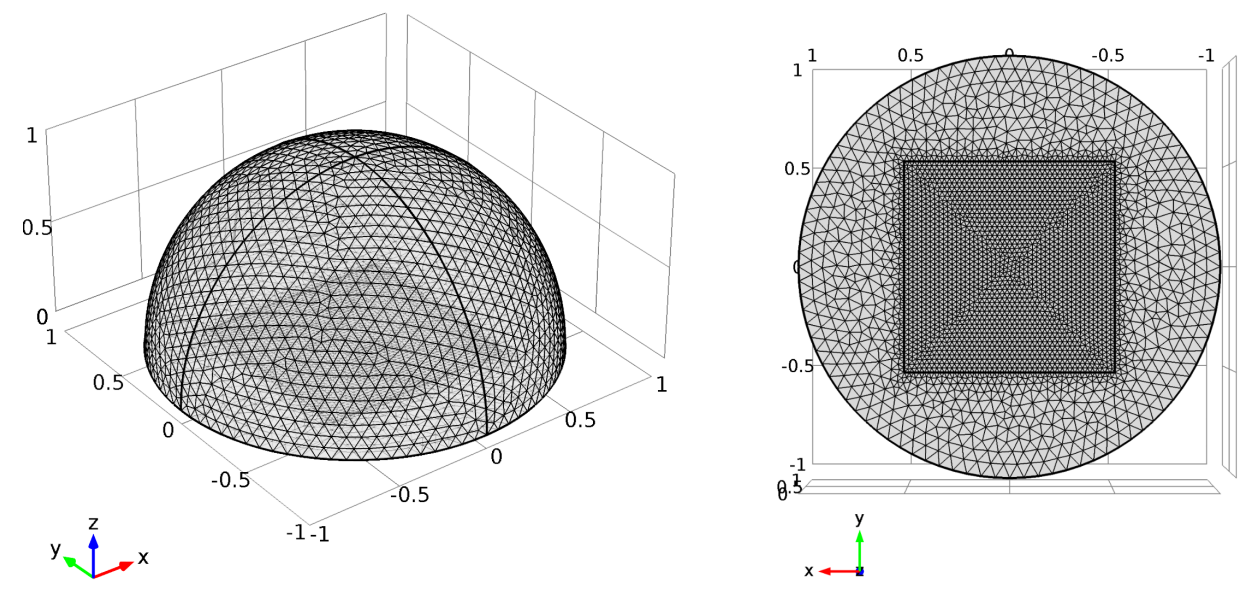

Figure 3: Used mesh for the plate radiating sound into the free field. The plate borders are indicated with the thick line on the right hand side of the figure (shown at $z=0 \mathrm{~m}$ ).

\begin{tabular}{ccc} 
Properties & Symbol & Value \\
\hline Young's modulus & $E$ & $69 e 9 \mathrm{~Pa}$ \\
Poisson's ratio & $\nu$ & 0.3 \\
Density & $\rho$ & $2700 \mathrm{~kg} / \mathrm{m}^{3}$ \\
Thickness & $t$ & $0.003 \mathrm{~m}$ \\
Dimensions & $l, b$ & $(1,1) \mathrm{m}$ \\
Damping parameters & $\alpha, \beta$ & $25,2.5 e-7$ \\
\hline
\end{tabular}

Table 2: Physical properties of the aluminum plate.

plate and the chosen infinite elements are mapped conjugated Astley-Leis elements with an order of 4. This leads to a total amount of 36201 DOFs. The resulting mesh is shown from different perspectives in Fig. 3.

\subsubsection{Model order reduction procedure}

As has already been indicated in Sec. 3.4, the model order reduction process for these examples is done by using the SOAR algorithm. Furthermore, an iterative procedure is used, called the Iterative Rational Krylov Approximation (IRKA), to find a reduced model that is $H_{2}$ optimal [11]. This algorithm runs multiple instances of the SOAR procedure and tunes the expansion points of the Krylov subspace until convergence is reached. Since the system has a second order form, the standard IRKA procedure cannot be applied directly, since it would lead to a doubling of the expansion points at every IRKA iteration [10]. Therefore the Second Order IRKA (SO-IRKA) has been used, see [10] for details of the implementation. The accuracy of 

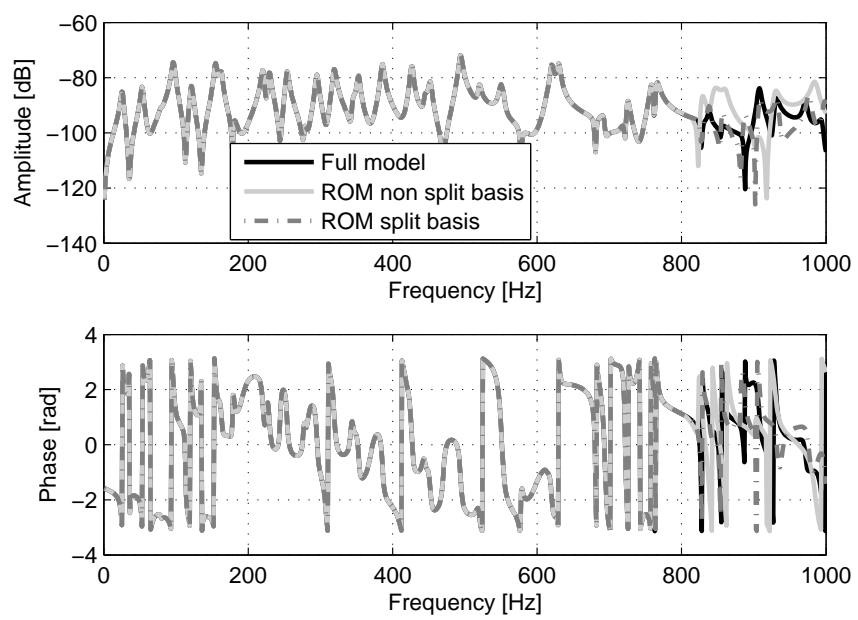

Figure 4: Comparison of FRFs between the full model, the reduced model calculated with an unsplit basis, and the reduced model calculated with a split basis. For both models the $u-\phi$ formulation is used.

the ROM is found by calculating the relative error between a reference model (the full system) and the ROM, which is defined as

$$
\varepsilon(\omega)=\frac{|\hat{G}(\omega)-G(\omega)|}{|G(\omega)|}
$$

in which $\hat{G}(\omega)$ is the transfer function of the ROM and $G(\omega)$ is the transfer function of the full model. The initial value for the dimensions of the reduced basis is set to $k=200$. This value is chosen, because it leads to a clear comparison between the different formulations. Both the ROM with a split basis (Sec. 3.4.1) and the non split basis (Sec. 3.4.2) are used to calculate the ROM. For the split basis this means that approximately three times the number of states (600) are acquired, as compared to method in which the bases are non split. The plate is excited by a point force of $1 \mathrm{~N}$ at the point $(0.3992,0.3686,0)$ in the $z$-direction. The response is calculated in the acoustic domain at coordinate $(0.4153,0.1614,0.4811)$. The FRF of the full system and the ROM calculated with SO-IRKA are shown in Fig. 4.

The relative error between the full model and both MOR schemes is shown in Fig. 5. It can be seen that the MOR resulting from a split basis gets more accurate results, but again it has to be remarked here that due to the split basis, the amount of DOFs used for this model are approximately three times as many as that of the ROM with the non split basis. 


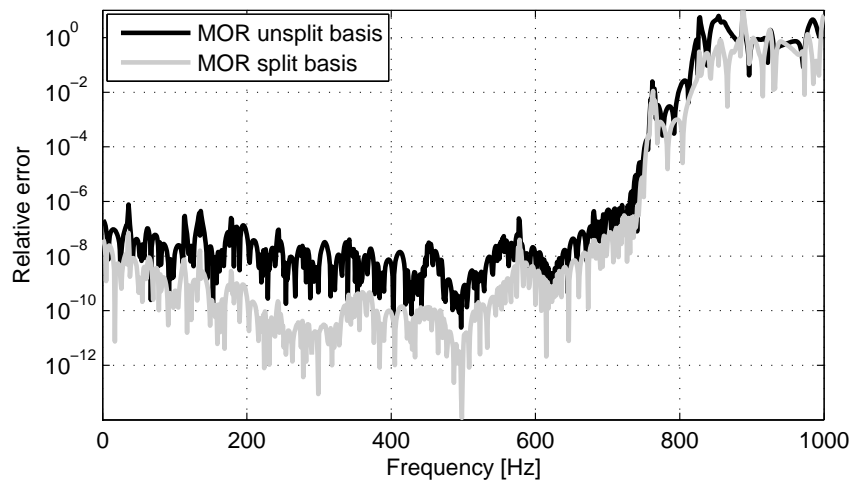

Figure 5: Relative error between the full model and two MOR schemes.

The stability of the reduced system can be assessed by evaluating the generalized eigenvalues resulting from the generalized eigenvalue problem $(A, E)$ of the full and the reduced system. Furthermore, the eigenvalues between the ROM before and after transformation to state space form are assessed. This comparison is made in Fig. 6 for the reduced model in the $u-\phi$ form with the unsplit basis. All eigenvalues are smaller than zero, so they conform to the stability requirements [3]. Also, the eigenvalues until approximately $5000 \mathrm{rad} / \mathrm{s}$ of the full and reduced model match really well, which indicates that the ROM should be accurate until approximately $795 \mathrm{~Hz}$. It can indeed be seen in the FRFs in Fig. 4 that the relative error increases to approximately $100 \%$ for frequencies higher than $795 \mathrm{~Hz}$. Between the two reduced systems there is no noticeable difference between the eigenvalues, confirming the statement in Sec. 4.2 that the eigenvalues should not change while applying the transformation in Eq. (38).

An eigenvalue analysis of the unsplit ROM with the $u-p$ formulation is shown in Fig. 7. It can be seen that multiple eigenvalues lie on the positive real part of the figure. This means that the system is unstable, as predicted in Sec. 3.3. Therefore, for further analysis the system with the $u-p$ will not be taken into account.

\subsubsection{Time domain analysis}

For the time domain analysis a band-limited impulse is chosen that is acquired by filtering a discrete pulse with a low-pass filter, tuned to have a pass band until $600 \mathrm{~Hz}$. A sampling frequency of $5 \mathrm{kHz}$ is chosen. The band-limited pulse can be seen in Fig. 8. All initial conditions are set to zero. Firstly, the full model is compared to the reduced model with Newmark- $\beta$ time integration, see Fig. 9, and the relative error is plotted in the bottom 


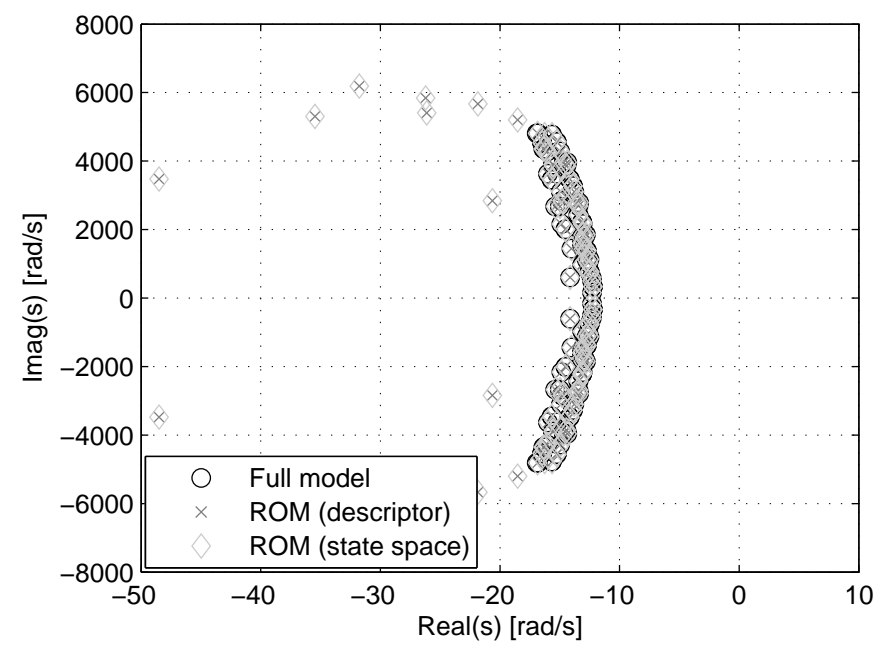

Figure 6: Generalized eigenvalue comparison between the full system and the reduced system, reduced with an unsplit basis $(u-\phi)$, before and after transformation to state space format.

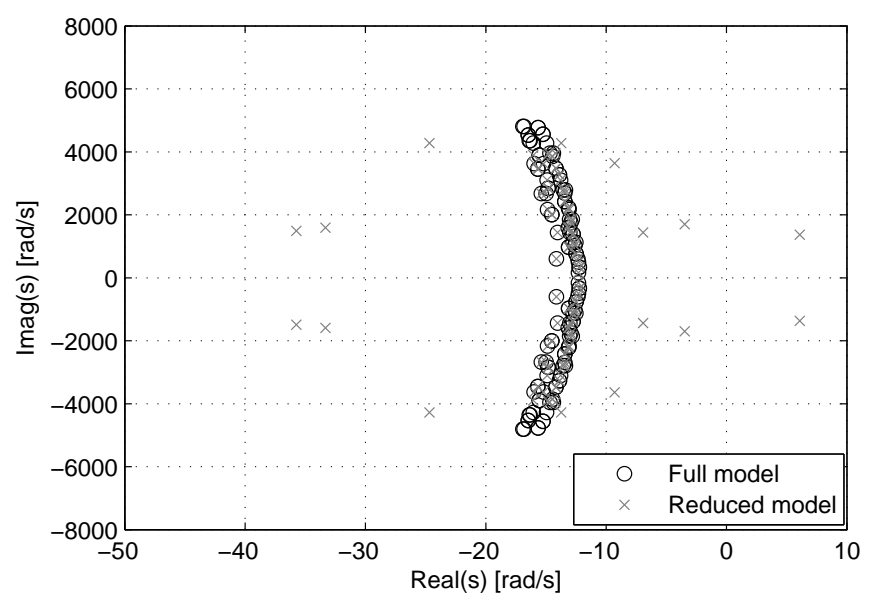

Figure 7: Generalized eigenvalue comparison between the full system and the reduced system, reduced with an unsplit basis $(u-p)$. 


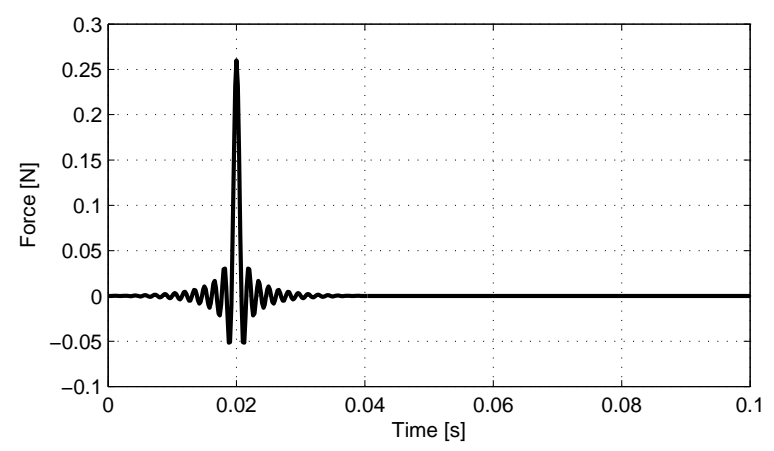

Figure 8: Force as function of time.

part of the figure. The average acceleration method $(\gamma=1 / 2, \beta=1 / 4)$ is used. It can be seen that the MOR gives a good approximation of the full system and is stable. Secondly, a comparison is made between the response of the Newmark- $\beta$ integrator of the full system and the exponential integrator, see Fig. 10. In this case there is a significant difference between the two signals. This can be explained by the period error that is characteristic for implicit time integrators [1]. To further elaborate on this, the FRFs corresponding to the time domain signals were calculated to check the frequency content of both responses. The result can be seen in Fig. 11, where they are compared to the FRF of the full system. It can be seen that for low frequencies both integrators give similar responses, but that for increasing frequencies the resonance peaks of the Newmark- $\beta$ scheme are shifted as compared to the full system, while the resonance peaks of the ROM with the exponential integrator are at the same position. The exponential integrator clearly outperforms the Newmark- $\beta$ integrator. To conclude the first example, a plot was made of the radiated sound for both the full model and the ROM at two time instances, and furthermore of the absolute value of the error between the two models, see Fig. 12. This data can be post-processed to obtain further physical quantities of interest, such as the radiation efficiency of the plate [32].

\subsection{Model 2: Vibro-acoustic analysis of a loudspeaker}

For the second model the performance of the proposed algorithm is analyzed on a more geometrically complex model, which is a loudspeaker radiating sound into the free field. A CAD drawing of the loudspeaker is shown in Fig. 13. It can be seen that the loudspeaker consists of a woofer, tweeter and a bass reflex port. The box dimensions are $(0.663,0.395,0.400) \mathrm{m}$, the diameter of the tweeter is $0.125 \mathrm{~m}$, the diameter of the woofer is $0.250 \mathrm{~m}$ 

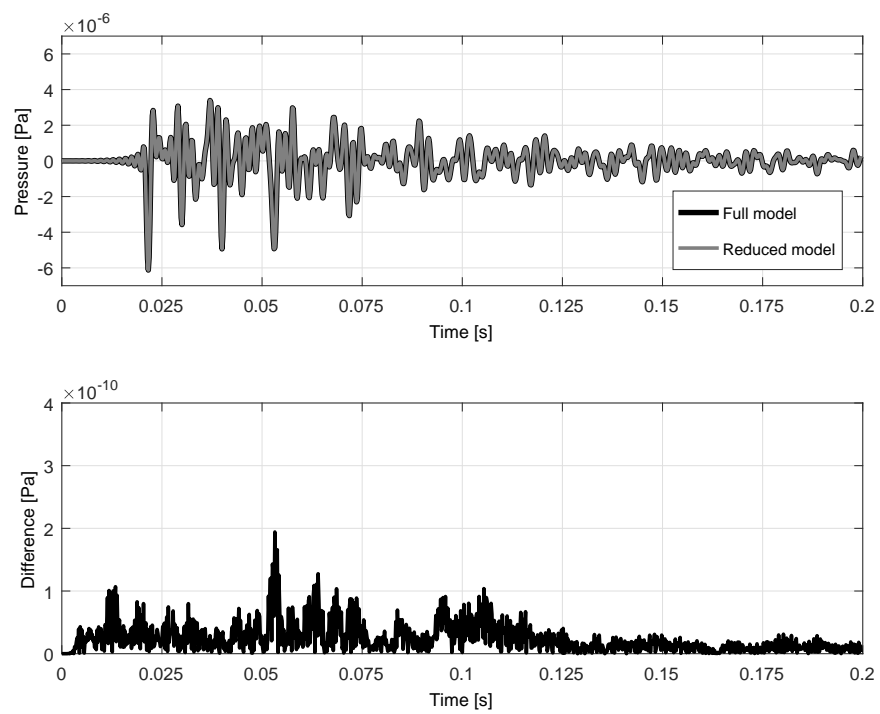

Figure 9: Comparison of results from Newmark- $\beta$ for the full model and the reduced model ( $\mathrm{u}-\phi$ formulation) at the top, and the difference between the two models at the bottom.
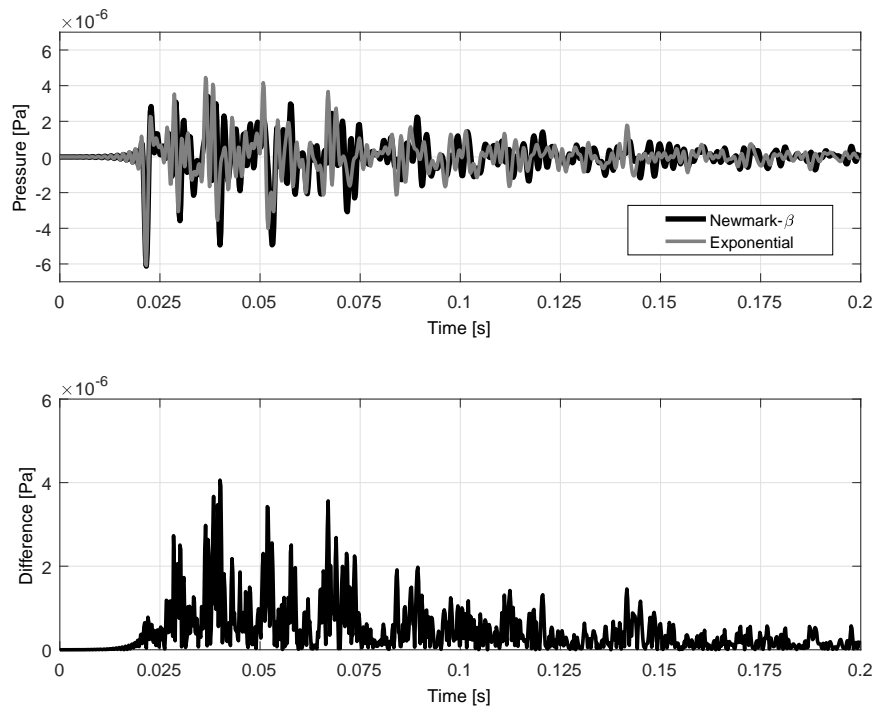

Figure 10: Comparison of results from Newmark- $\beta$ for the full model and exponential integration of the reduced model, and the difference between the two models at the bottom. 

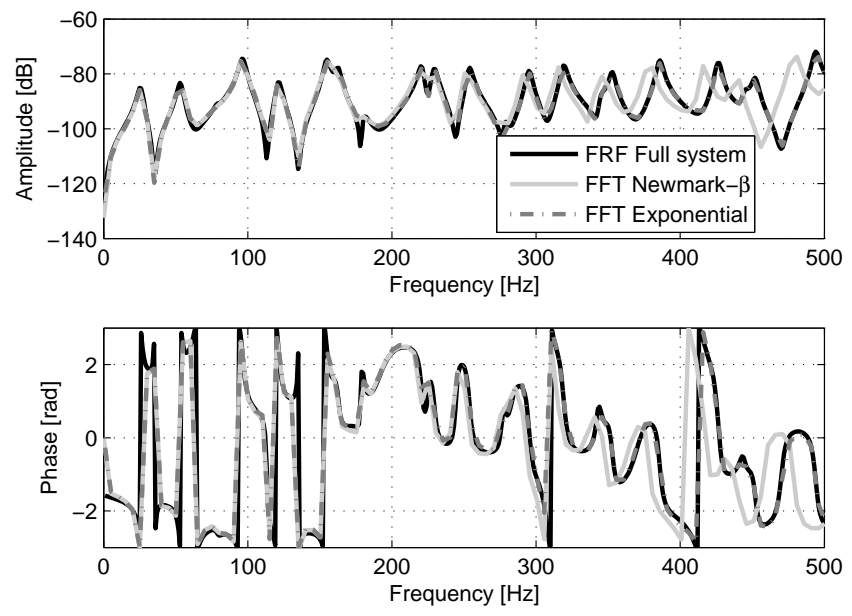

Figure 11: Comparison of the FRF of the full model with the Fourier transformed output signals of the Newmark- $\beta$ and exponential scheme, resulting from the band-limited impulse signal shown in Fig. 8.
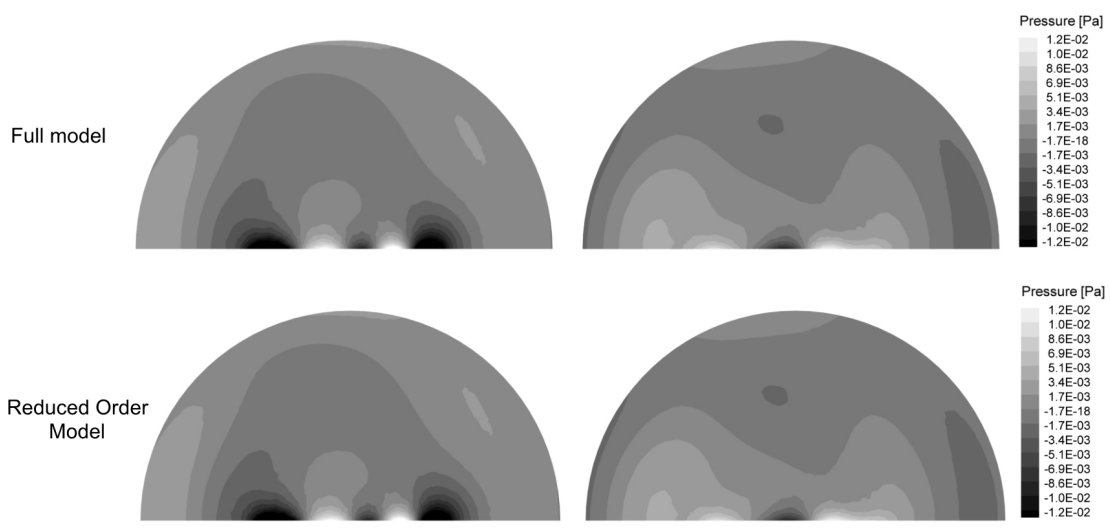

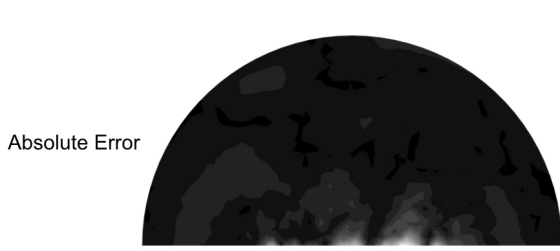

$t=0.020 s$

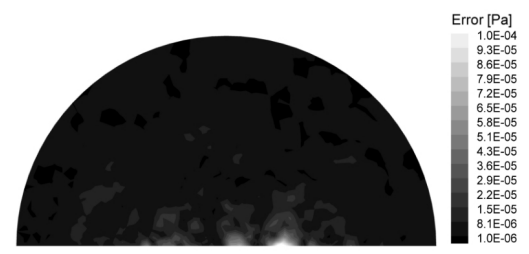

$\mathrm{t}=0.040 \mathrm{~s}$

Figure 12: Acoustic pressure comparison at $x=0$ for the full model and the ROM at $t=0.020 \mathrm{~s}$ and $t=0.040 \mathrm{~s}$. 


\begin{tabular}{ccc} 
Variable & Symbol & Value \\
\hline Young's modulus & $E$ & $69 \mathrm{GPa}$ \\
Poisson's ratio & $\nu$ & 0.3 \\
Density (Speaker) & $\rho$ & $2700 \mathrm{~kg} / \mathrm{m}^{3}$ \\
Thickness & $t$ & $0.25 \mathrm{~mm}$ \\
Proportional damping & $\alpha, \beta$ & $20,10^{-5}$ \\
\hline
\end{tabular}

Table 3: Material properties.

and the bass reflex port has a length of $0.242 \mathrm{~m}$ and a diameter of $0.1 \mathrm{~m}$. Both the woofer and the tweeter are modeled in the structural domain with linear triangular FE shell elements and are simply supported at the edges. The rest of the speaker box is considered rigid and is modeled by a sound hard boundary condition in the acoustic domain. The interior and exterior of the box are both modeled with linear tetrahedral elements within a sphere of $1 \mathrm{~m}$ diameter and the origin of the sphere is placed in the middle of the box at point $(0.0,-0.15-0.2)$. The acoustic element size has been chosen such that a minimum of 6 elements per wavelength is present at $f=500 \mathrm{~Hz}$ (which gives an element size of less than $0.1 \mathrm{~m}$ ). The same acoustic properties as in Model 1 have been used, so $c=343 \mathrm{~m} / \mathrm{s}$, and $\rho=1.225 \mathrm{~kg} / \mathrm{m}^{3}$. For the structural mesh, the element size is significantly finer than 6 elements per wavelength, since the complex geometry of the woofer and tweeter requires a fine mesh (an element size of approximately $0.008 \mathrm{~m}$ for the woofer and the outside of the tweeter and approximately $0.003 \mathrm{~m}$ for the inside of the tweeter). Infinite elements with a radial order of 6 are placed at the edge of the sphere to simulate the Sommerfeld radiation condition. This leads to a system consisting of 25227 DOFs, of which 8846 structural DOFs, 14226 acoustic FE DOFs, and 2155 acoustic IE DOFs. The material properties of the woofer and tweeter are given in Table 3.

\subsubsection{Frequency domain comparison}

A point force is applied at coordinate $(-0.0108,-0.0073,0.0125)$ on the woofer and the output point is chosen above the woofer at $(0.0849,-0.07,0.0173)$. Again the FRFs of both the reduced models with a split basis and the unsplit basis are compared, as shown in Fig. 14 and Fig. 15. This is done for a reduced model of 75 DOFs for the unsplit ROM. Also here the IRKA procedure has been applied to iteratively find an $\mathrm{H}_{2}$ optimal ROM. Since the FRF has less resonance peaks, this ROM can actually be build up with less DOFs to accurately describe the system. With the chosen model size, the FRF is accurate up until $800 \mathrm{~Hz}$. Since the mesh is designed to give only valid results until $500 \mathrm{~Hz}$ one could consider lowering the amount of DOFs 

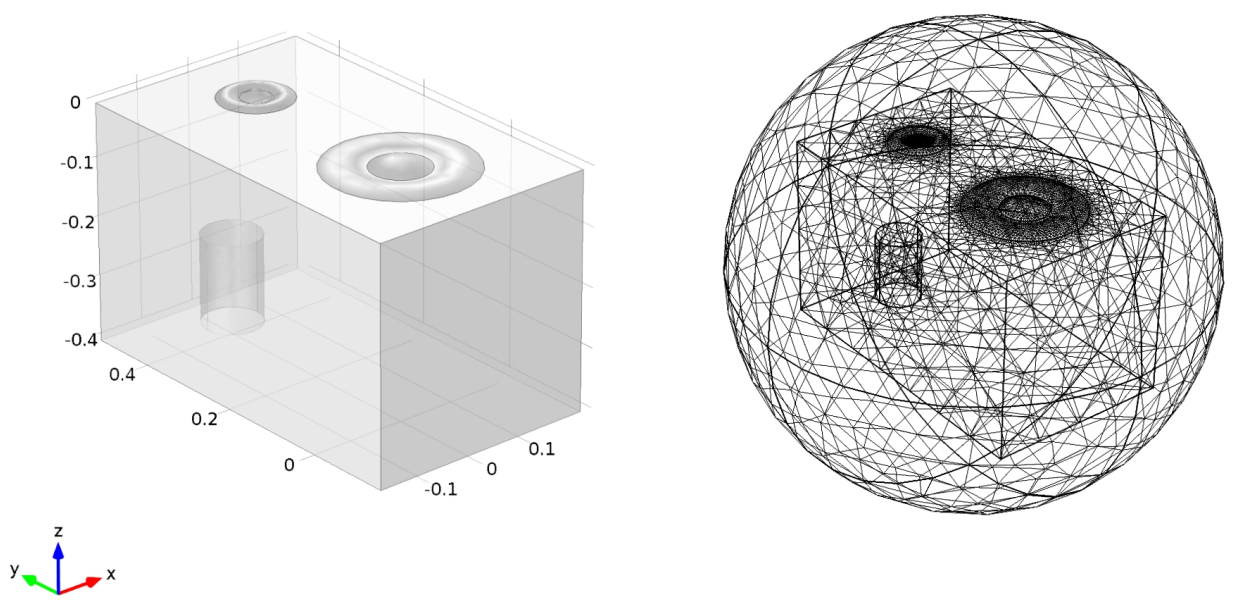

Figure 13: CAD drawing of a loudspeaker with dimensions in meters (left) and the finite element mesh (right).

of the ROM if it is going to be used in a real-time application, but for this example the model of 75 DOFs will be used. The eigenvalues of the full system and the reduced system (unsplit) before and after transformation to state space form are shown in Fig. 16 and the conclusions are similar to the model described in Sec. 5.1. The ROM matches the eigenvalues well until approximately $5000 \mathrm{rad} / \mathrm{s}$, which corresponds to a frequency of $796 \mathrm{~Hz}$. This is also the point in which the relative error between the full model and the ROM increases. There is no significant difference between the eigenvalues of the ROM before and after transformation to state space form.

\subsection{Time domain response}

Similar to model 1, a band-limited input signal is chosen, but now until $500 \mathrm{~Hz}$. Again the sampling frequency is chosen at $5000 \mathrm{~Hz}$ and for the comparison between the full model and the ROM in the first instance a Newmark- $\beta$ integrator is used. The result is shown in Fig. 17. Also here the results are compared to an exponential integrator and this is shown in Fig. 18.

Finally, the time domain response in the whole acoustic field is compared for the full and reduced model at two time instances. The resulting contour plots at $x=0$ are shown in Fig. 19. For both the full model and the ROM a Newmark- $\beta$ scheme is used. It can be seen that absolute value of the error in the reduced order model is similar to the results in Fig. 17. 

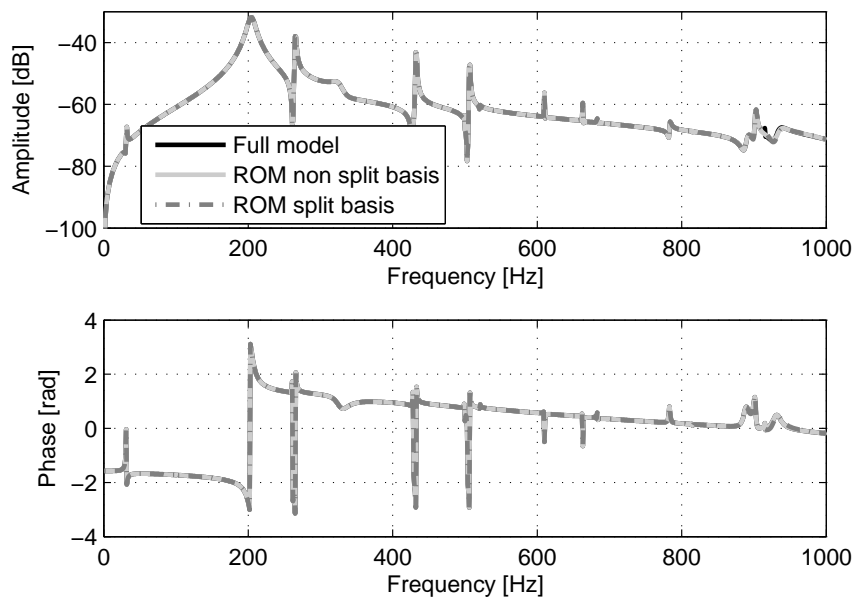

Figure 14: Comparison of FRFs between full model, the reduced model calculated with an unsplit basis and the reduced model calculated with a split basis.

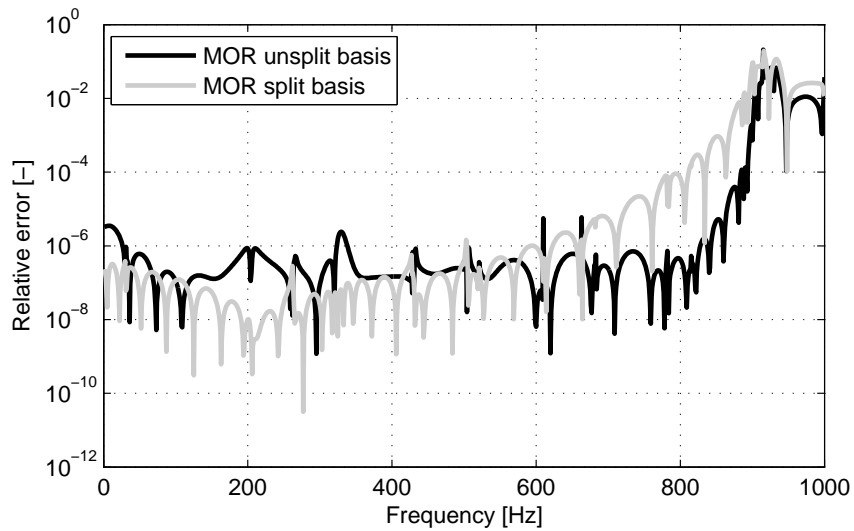

Figure 15: Relative error between the FRF of the full model and the two MOR schemes. 


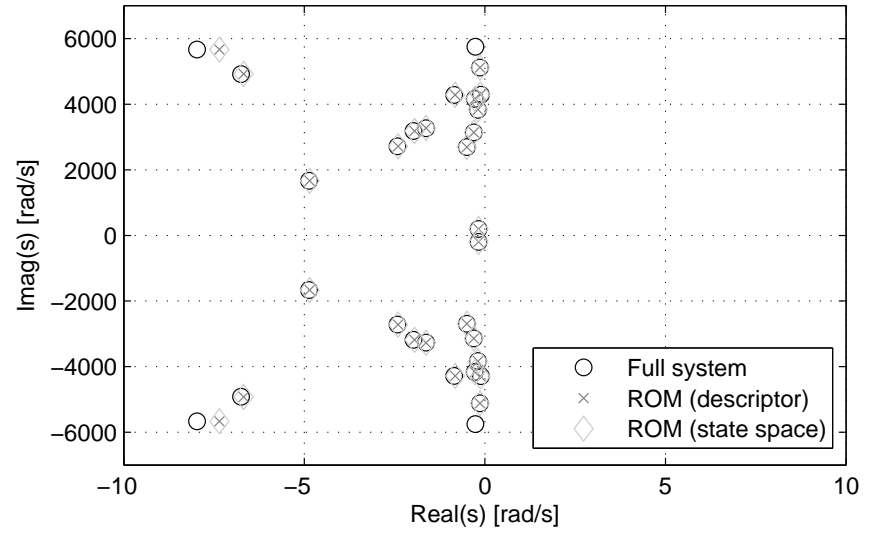

Figure 16: Comparison of the eigenvalues of the full system with the ROM in descriptor form and state space form.
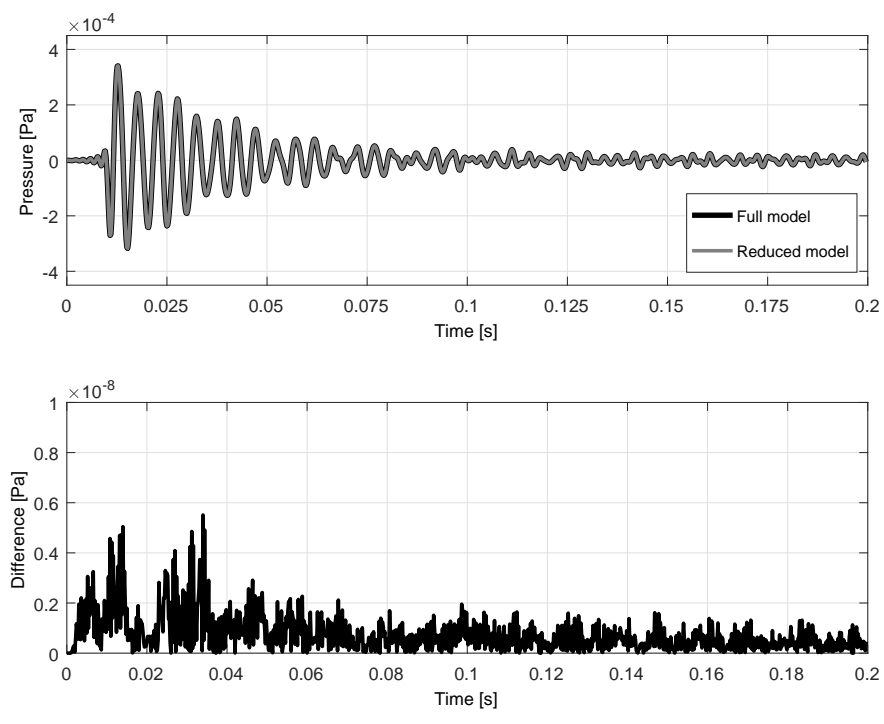

Figure 17: Comparison of the results from Newmark- $\beta$ for the full model and the reduced model at the top and the difference between the two models at the bottom. 

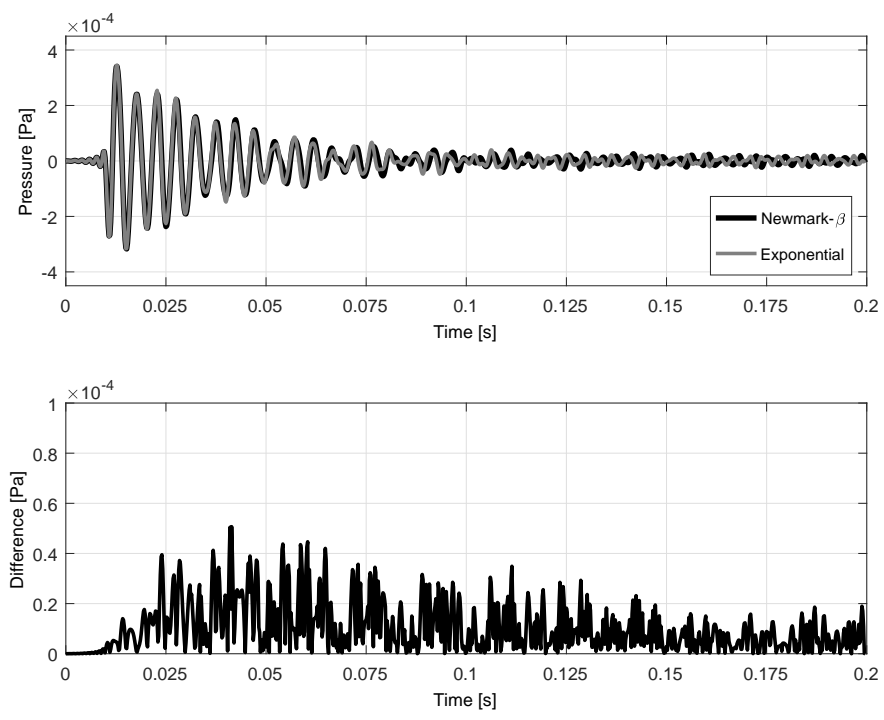

Figure 18: Comparison of the results from Newmark- $\beta$ for the full model and the exponential integration of the reduced model at the top and the difference between the two models at the bottom.

\section{Conclusions and recommendations}

In this paper a method for reducing the model size of exterior time domain vibro-acoustic problems modeled with the finite element method is derived. The derived method is shown to preserve the stability of the original system and is shown to give accurate results with only a fraction of the amount of degrees of freedom of the original system. The reduction scheme uses a onesided projection, performed with a congruence transform. Infinite elements are used to account for the Sommerfeld radiation condition. For the implementation of the infinite elements the conjugated Astley-Leis formulation is chosen, since it leads to frequency independent system matrices.

Two approaches are used for the reduction of the system matrices. Firstly, a strategy in which the calculated reduction basis is split into three different domains (structural, acoustic FE, and acoustic IE). This leads to larger reduced models, but can be used on both the system matrices in the $u-p$ and the $u-\phi$ formulation. Secondly, a strategy in which the calculated reduction basis is not split. This leads to smaller reduced models for the same accuracy, but is not guaranteed to be stable. It is shown that a modified version of the $u-\phi$ formulation leads to a stable reduced order model for the non split basis and is thus the preferred implementation. 


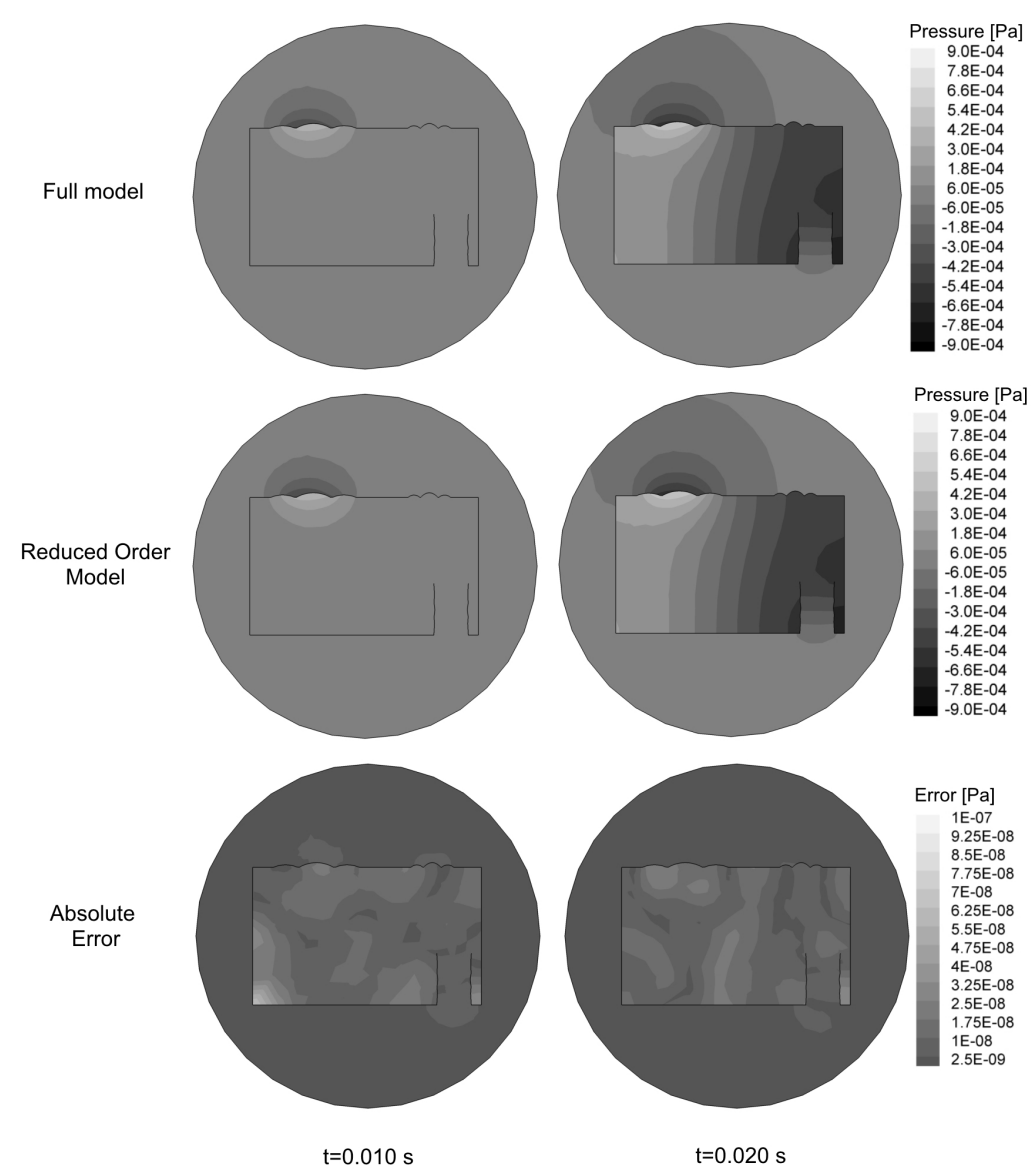

Figure 19: Acoustic pressure comparison at $x=0$ for the full model and the ROM at $t=0.01 \mathrm{~s}$ and $t=0.020 \mathrm{~s}$. 
For an efficient way of time-integrating the ROM, a state space format of the system is preferred, since then an explicit exponential integration scheme is applicable. This means that the mass matrix has to be inverted. Since the addition of infinite elements might lead to a singular mass matrix, this is not always possible. Therefore, a method is described that avoids this inversion by using an SVD-decomposition of the $E$-matrix. This leads to the desired state space description, which can be discretized by using the exponential integrator.

The described method is tested with two numerical models. The first model analyzes the vibro-acoustic behavior of a plate radiating sound into the free field. The second model considers the geometrically more complex system of a loudspeaker. The accuracy of the method is assessed in both the frequency and time domain. It is shown that the proposed method gives time stable ROMs with a high accuracy for only a fraction of the DOFs of the full system. Furthermore, it is shown that the described procedure for transforming the system from a descriptor form to state space form does not alter the finite eigenvalues, thus also not the stability of the system. The comparison between the time responses resulting from the use of a Newmark- $\beta$ scheme of the full system and the reduced system show that the two give very similar results. When the exponential integrator is used, the response is slightly different from Newmark- $\beta$ but this can be explained by plotting the FRFs calculated by doing a Fourier transform of the response and excitation. It can be seen that the resonance peaks in the FRF of the Newmark- $\beta$ scheme are slightly shifted for higher frequencies. Thus the derived method for model order reduction of exterior vibro-acoustic finite element models provides an effective way to arrive at a time stable, accurate, reduced order model that can be time-integrated in an efficient manner. This paves the way to applications in which (near-)real time simulations are required, such as model-based structural health monitoring or active structural/acoustic control.

\section{Acknowledgments}

The research of S. van Ophem is funded by an Early Stage Researcher grant within the European Project ANTARES Marie Curie Initial Training Network (GA 606817). The research of E. Deckers is funded by a grant from the Fund for Scientific Research, Flanders (F.W.O). The Research Fund KU Leuven is gratefully acknowledged for its support. The computational resources and services used in this work were provided by the VSC (Flemish Supercomputer Center), funded by the Research Foundation, Flanders (FWO) and the Flemish Government, department EWI. 


\section{Appendix A.}

Here a proof is given of Lemma 1:

Proof. Consider the generalized eigenvalue problem that results from a descriptor system:

$$
A z=\lambda E z .
$$

Let $\lambda \in \mathbb{C}$ be an eigenvalue with $\lambda=\mu+i \nu$ and $\mu, \nu \in \mathbb{R}$. Define the corresponding right eigenvector as $z \in \mathbb{C}^{n}$ with $z=x+i y$, where $x, y \in \mathbb{R}^{n}$. Substitution gives:

$$
A(x+i y)=(\mu E+i \nu E)(x+i y) .
$$

Eq. (A.2) is split into two equations, representing the real and imaginary part, which are premultiplied with $x^{T}$ and $y^{T}$ respectively:

$$
\begin{aligned}
x^{T} A x & =\mu x^{T} E x-\nu x^{T} E y, \\
y^{T} A y & =\mu y^{T} E y+\nu y^{T} E x .
\end{aligned}
$$

Rewriting and summing the two equations gives:

$$
x^{T} A x+y^{T} A y-\mu x^{T} E x-\mu y^{T} E y=\nu\left(x^{T} E y-y^{T} E x\right)=0 .
$$

This gives

$$
\mu=\frac{x^{T} A x+y^{T} A y}{x^{T} E x+y^{T} E y} \leq 0 \text { or } \infty .
$$

Infinite eigenvalues appear when the descriptor system is singular. These eigenvalues result from algebraic conditions in the system of differential equations and when treated properly, these eigenvalues do not pose problems with instabilities, as shown in in Sec. 4. Therefore, it is proven that the lemma holds.

\section{Appendix B.}

Here a proof is given of Lemma 2: 
Proof. The matrix $E$ is a block diagonal matrix, of which both terms on the diagonal are semi-positive definite, thus the matrix as a whole will be semi-positive definite, $E \geq 0$. The properties of matrix $A$ will be assessed as follows:

$$
\begin{aligned}
\mathfrak{R e}\left\{x^{T} A x\right\} & =\left[\begin{array}{ll}
x_{1}^{T} & x_{2}^{T}
\end{array}\right]\left[\begin{array}{cc}
0 & K^{T} \\
-K & -C
\end{array}\right]\left[\begin{array}{l}
x_{1} \\
x_{2}
\end{array}\right] \\
& =-x_{2}^{T} C x_{2}+x_{1}^{T} K^{T} x_{2}-x_{2}^{T} K x_{1} \\
& =-x_{2}^{T} C x_{2}+x_{2}^{T} K x_{1}-x_{2}^{T} K x_{1} \\
& =-x_{2}^{T} C x_{2} \leq 0,
\end{aligned}
$$

together with the results of Lemma 1 this concludes the proof of this lemma.

\section{Appendix C.}

The bounded-input bounded-output stability of a state space system is determined by the eigenvalues of the matrix $A$, which are required to have a real part smaller than zero. Suppose that this is the case and that the matrix is of full rank (no repeated eigenvalues). Thus,

$$
\lambda_{1}<\lambda_{2}<\ldots .<\lambda_{n}<0,
$$

The following matrix exponential will be used, with $T_{s}$ defined as the sampling period:

$$
e^{A T_{s}},
$$

Now the following condition holds for every eigenvalue ( $\mathrm{v}$ is the corresponding eigenvector)

$$
e^{A T_{s}} \mathbf{v}=e^{\lambda T_{s}} \mathbf{v},
$$

This implies that for the matrix resulting from the calculation of the matrix exponential the new eigenvalues will be exponentials of the eigenvalues in matrix $A$. For discrete systems the eigenvalues should be within the unit circle in the z-plane to give a stable system. Since the exponential of a number smaller than zero is smaller than 1 and the imaginary part does not influence the amplitude, this will be the case. Proof for a random complex number $a+i b$ 


$$
\begin{aligned}
e^{a+i b} & =e^{a} e^{i b} \\
& =e^{a}(\cos b+i \sin b) .
\end{aligned}
$$

Here Euler's formula is used. The magnitude of $(\cos b+i \sin b)$ is equal to one. Thus if $a$ is smaller than zero (which is true for stable eigenvalues in continuous time) this results in a magnitude smaller than one, thus inside the unit circle in the z-domain.

[1] R. D. Cook, D. S. Malkus, M. E. Plesha, R. J. Witt, Concepts and Applications of Finite Element Analysis, fourth edition Edition, Wiley, Madison, 2002.

[2] O. von Estorff, Boundary Elements in Acoustics: Advances and Applications, WITpress, 2000.

[3] G. Duan, Analysis and Design of Descriptor Linear Systems, SpringerVerlag, New York, 2010.

[4] B. Moore, Principal component analysis in linear systems: Controllability, observability, and model reduction, IEEE Transactions on Automatic Control 26 (1) (1981) 17-32. doi:10.1109/TAC.1981.1102568.

[5] B. Salimbahrami, Structure preserving order reduction of large scale second order models, PhD thesis, Technische Universität München (2005).

[6] E. J. Grimme, Krylov Projection Methods for Model Reduction, Ph.D. thesis, University of Illinois, Illinois (1997).

[7] Z. Bai, Y. Su, Dimension Reduction of Large-Scale Second-Order Dynamical Systems via a Second-Order Arnoldi Method, SIAM Journal on Scientific Computing 26 (5) (2005) 1692-1709. doi:10.1137/040605552. URL http://epubs.siam.org/doi/abs/10.1137/040605552

[8] Z. Bai, Y. Su, SOAR: A Second-order Arnoldi Method for the Solution of the Quadratic Eigenvalue Problem, SIAM Journal on Matrix Analysis and Applications 26 (3) (2005) 640-659. doi:10.1137/S0895479803438523.

URL http://epubs.siam.org/doi/abs/10.1137/S0895479803438523

[9] R.-c. Li, Z. Bai, Structure-preserving model reductions using a Krylov subspace projection formulation, Communications in Mathematical Sciences 3 (2) (2005) 179-199. doi:10.4310/CMS.2005.v3.n2.a6.

URL http://intlpress.com/site/pub/pages/journals/items/cms/content/vols/0003 
[10] S. Wyatt, Issues in Interpolatory Model Reduction: Inexact Solves, Second-order Systems and DAE's, Ph.D. thesis, Blacksburg, Virginia (2012).

[11] S. Gugercin, A. C. Antoulas, C. A. Beattie, A Rational Krylov Iteration for Optimal $\mathrm{H}_{2}$ Model Reduction, in: Proceedings of the 17th International Symposium on Mathematical Theory of Networks and Systems, Japan, 2006, pp. 1665-1667.

[12] A. van de Walle, F. Naets, E. Deckers, W. Desmet, Stability-preserving model-order reduction for time-domain simulation of vibro-acoustic FE models, International Journal for Numerical Methods in Engineering 109 (6) (2016) 889-912. doi:10.1002/nme.5323.

URL http://onlinelibrary.wiley.com/doi/10.1002/nme.5323/full

[13] J. B. Keller, D. Givoli, Exact non-reflecting boundary conditions, Journal of Computational Physics 82 (1) (1989) 172-192. doi:10.1016/00219991(89)90041-7.

URL http://www.sciencedirect.com/science/article/pii/0021999189900417

[14] J. P. Berenger, A perfectly matched layer for the absorption of electromagnetic waves, Journal of Computational Physics 114 (2) (1994) 185-200. doi:10.1006/jcph.1994.1159.

URL http://www. sciencedirect.com/science/article/pii/S0021999184711594

[15] B. Engquist, A. Majda, Absorbing Boundary Conditions for the Numerical Simulation of Waves, Mathematics of Computation 31 (139) (1977) 629-651. doi:10.2307/2005997.

URL http://www.jstor.org/stable/2005997

[16] R. J. Astley, G. J. Macaulay, J. P. Coyette, L. Cremers, Threedimensional wave-envelope elements of variable order for acoustic radiation and scattering. Part I. Formulation in the frequency domain, The Journal of the Acoustical Society of America 103 (1) (1998) 49-63. doi:10.1121/1.421106.

URL http://scitation.aip.org/content/asa/journal/jasa/103/1/10.1121/1.421106

[17] R. J. Astley, J. P. Coyette, L. Cremers, Three-dimensional waveenvelope elements of variable order for acoustic radiation and scattering. Part II. Formulation in the time domain, The Journal of the Acoustical Society of America 103 (1) (1998) 64-72. doi:10.1121/1.421107. URL http://scitation.aip.org/content/asa/journal/jasa/103/1/10.1121/1.421107 
[18] R. J. Astley, J. A. Hamilton, The stability of infinite element schemes for transient wave problems, Computer Methods in Applied Mechanics and Engineering 195 (29-32) (2006) 3553-3571. doi:10.1016/j.cma.2005.01.026.

URL http://www.sciencedirect.com/science/article/pii/S0045782505002446

[19] D. S. Burnett, A three-dimensional acoustic infinite element based on a prolate spheroidal multipole expansion, The Journal of the Acoustical Society of America 96 (5) (1994) 2798-2816. doi:10.1121/1.411286.

URL http://scitation.aip.org/content/asa/journal/jasa/96/5/10.1121/1.411286

[20] B. Kaltenbacher, M. Kaltenbacher, I. Sim, A modified and stable version of a perfectly matched layer technique for the 3-d second order wave equation in time domain with an application to aeroacoustics, Journal of Computational Physics 235 (2013) 407-422. doi:10.1016/j.jcp.2012.10.016.

URL http://www. sciencedirect.com/science/article/pii/S0021999112006055

[21] R. J. Astley, Infinite elements for wave problems: a review of current formulations and an assessment of accuracy, International Journal for Numerical Methods in Engineering 49 (7) (2000) 951-976. doi:10.1002/1097-0207(20001110) 49:7<951: : AID-NME989>3 . 0 . CO;2-T. URL http://onlinelibrary . wiley.com/doi/10 .1002/1097-0207(20001110) 49:7<951:

[22] R. J. Astley, W. Eversman, Finite element formulations for acoustical radiation, Journal of Sound and Vibration 88 (1) (1983) 47-64. doi:10.1016/0022-460X(83)90678-8.

URL http://www.sciencedirect.com/science/article/pii/0022460X83906788

[23] D. Dreyer, O. von Estorff, Improved conditioning of infinite elements for exterior acoustics, International Journal for Numerical Methods in Engineering 58 (6) (2003) 933-953. doi:10.1002/nme.804. URL http://onlinelibrary.wiley.com/doi/10.1002/nme.804/abstract

[24] R. J. Astley, Infinite Elements, in: S. Marburg, B. Nolte (Eds.), Computational Acoustics of Noise Propagation in Fluids: Finite and Boundary Element Methods, Springer, 2008, pp. 197-230.

[25] O. von Estorff, S. Petersen, D. Dreyer, Efficient Infinite Elements based on Jacobi Polynomials, in: S. Marburg, B. Nolte (Eds.), Computational Acoustics of Noise Propagation in Fluids: Finite and Boundary Element Methods, Springer, 2008, pp. 231-250. 
[26] J. Cipolla, Acoustic infinite elements with increased robustness, in: Proceedings of ISMA 2002, Leuven, Belgium, 2002, pp. 2181-2188.

[27] G. C. Everstine, A symmetric potential formulation for fluid-structure interaction, Journal of Sound and Vibration 79 (1) (1981) 157-160. doi:10.1016/0022-460X(81)90335-7.

URL http://www.sciencedirect.com/science/article/pii/0022460X81903357

[28] R. W. Freund, Structure-Preserving Model Order Reduction of RCL Circuit Equations, in: W. H. A. Schilders, H. A. v. d. Vorst, J. Rommes (Eds.), Model Order Reduction: Theory, Research Aspects and Applications, no. 13 in Mathematics in Industry, Springer Berlin Heidelberg, 2008, pp. 49-73, dOI: 10.1007/978-3-540-78841-6_3.

[29] S. Gugercin, T. Stykel, S. Wyatt, Model Reduction of Descriptor Systems by Interpolatory Projection Methods, SIAM Journal on Scientific Computing 35 (5) (2013) B1010-B1033. doi:10.1137/130906635.

URL http://epubs.siam.org/doi/abs/10.1137/130906635

[30] S. S. S. K. Kumar, Stability results for constrained dynamical systems, Ph.D. thesis, National University of Ireland Maynooth (Dec. 2012).

[31] A. W. Al-Mohy, N. J. Higham, A new scaling and squaring algorithm for the matrix exponential, SIAM Journal on Matrix Analysis and Applications 31 (3) (2009) 970-989. doi:10.1137/09074721X.

URL http://dx.doi.org/10.1137/09074721X

[32] C. E. Wallace, Radiation Resistance of a Rectangular Panel, The Journal of the Acoustical Society of America 51 (3B) (1972) 946-952. doi:10.1121/1.1912943.

URL http://asa.scitation.org/doi/abs/10.1121/1.1912943 